\title{
ON REGULAR SEMIGROUPS AND THEIR MULTIPLICATION
}

BY

PIERRE ANTOINE GRILLET

\begin{abstract}
A method is given for the construction of regular semigroups in terms of groups and partially ordered sets. This describes any regular semigroup $S$ and its multiplication by means of triples $(i, g, \lambda)$ with $i \in$ $S / \Re, \lambda \in S / \mathcal{E}$ and $g$ in the Schützenberger group of the corresponding $\Phi$-class. It is shown that the multiplication on $S$ is determined by certain simple products. Furthermore the associativity of these simple products implies associativity of the entire multiplication.
\end{abstract}

We give a general method for constructing arbitrary regular semigroups in terms of groups and partially ordered sets.

Let $S$ be a regular semigroup. Select a cross-section $\left(r_{i}\right)_{i \in I}$ of $\mathscr{R}$ (with $I=S / R$ ), a cross-section $\left(q_{\lambda}\right)_{\lambda \in \Lambda}$ of $\mathcal{L}$ (with $\Lambda=S / \mathscr{L}$ ), and one maximal subgroup of $S$ in each $\mathscr{D}$-class; the union of these subgroups is a set $G$. Each element of $S$ can then be put uniquely in the form $r_{i} g q_{\lambda}$, with $g \in G$ and $r_{i}, g$, $q_{\lambda}$ in the same $\mathscr{D}$-class. Our method gives a construction of $S$ in terms of the groups in $G$ and the partially ordered sets $I, \Lambda$, by showing how the multiplication of $S$ can be described under that form.

In $\S 1$, we define partial actions between the sets $G, I, \Lambda$, denoted by various symbols as seen below, and show that the multiplication of $S$ can be described in terms of these and three factor sets $\sigma, \tau, v$, by the formula

$$
(i, g, \lambda)(j, h, \mu)=\left(i+g(j * \lambda), \sigma_{i, g(j * \lambda)} g^{j * \lambda} \tau_{\lambda j} h_{\lambda * j} v_{(\lambda * j) h, \mu},(\lambda * j) h+\mu\right)
$$

where each $r_{i} g q_{\lambda}$ is now written as a triple $(i, g, \lambda)$.

Necessary and sufficient conditions for such a multiplication to define a regular semigroup are then given in \$3. The main difficulty here is the associativity of these multiplications; this is studied in $\$ 2$, where we prove an Associativity Lemma which is the deepest and most interesting part of the paper.

Although the result is, technically speaking, a structure theorem for arbitrary regular semigroups, it is still too technical to be very interesting as such. Forthcoming papers will show that it must be combined with more

Presented to the Society, January 16, 1974 under the title Regular semigroups; received by the editors February 24, 1975.

AMS (MOS) subject classifications (1970). Primary 20M10. 
elementary work and coextension concepts, to obtain a better view of the structure. Even without these applications, however, it shows that the most precise construction conceivable for general regular semigroups is possible; it also predicts the form of the multiplication, and tells us how to go about the construction. Most importantly, it takes care of the most difficult part of the construction, which is verifying that the complex multiplication thus obtained is associative. In some cases, the conditions for this become quite simple and the theorem can be applied directly also.

The author is indebted to Professors A. H. Clifford and M. P. Grillet for suggestions which somewhat shortened the proof of the Associativity Lemma.

\section{Structure data of a regular semigroup.}

1. In what follows, $S$ denotes a regular semigroup. Let $\left(D_{\alpha}\right)_{\alpha \in A}$ be the family of all $\mathscr{D}$-classes of $S$. In each $D_{\alpha}$, select an idempotent $e_{\alpha}$; denote by $G_{\alpha}$ the $\mathcal{H}$-class of $e_{\alpha}$, and by $G$ the union of all the groups $G_{\alpha}$ (in general not a subsemigroup of $S$ ). Let $I=S / R, \Lambda=S / \mathcal{L}$; denote the $\mathscr{R}$-class of $e_{\alpha}$ by $i_{\alpha}$, and its $\mathcal{L}$-class by $\lambda_{\alpha}$. When $i \in I, g \in G, \lambda \in \Lambda$, we write $i \equiv g$ to mean that $g \in S$ and the $\mathcal{R}$-class $i$ lie in the same $\mathscr{D}$-class; and similarly, $g \equiv \lambda$, $i \equiv \lambda$. Note that for each $i \in I$ there is a unique $i_{\alpha}$ such that $i \equiv i_{\alpha}$; and similarly for $G, \Lambda$ vs. the elements $e_{\alpha}, \lambda_{\alpha}$. Finally, for each $i \in I$ we select one $r_{i} \in S$ which lies in the $R$-class $i$ and satisfies $r_{i} \mathcal{L} e_{\alpha}$, where $\alpha$ is such that $i \equiv i_{\alpha}$; when $i=i_{\alpha}$, we pick $r_{i}=e_{\alpha}$. Similarly, for each $\lambda \in \Lambda$ we pick $q_{\lambda} \in \lambda$, with $q_{\lambda} \Re e_{\alpha}$ if $\lambda \equiv \lambda_{\alpha}, q_{\lambda}=e_{\alpha}$ if $\lambda=\lambda_{\alpha}$.

Assume $i \equiv e_{\alpha} \equiv \lambda$. Then $q_{\lambda} \Re e_{\alpha}, r_{i} \mathcal{E} e_{\alpha}$ shows that $e_{\alpha} q_{\lambda}=q_{\lambda}, r_{i} e_{\alpha}=r_{i}$; since also $e_{\alpha}=q_{\lambda} q_{\lambda}^{\prime}=r_{i}^{\prime} r_{i}$ for some $q_{\lambda}^{\prime}, r_{i}^{\prime} \in S$, the following result is immediate from Green's Lemma:

Proposition 1.1. For each $x \in S$, there exist unique $i \in I, \lambda \in \Lambda, g \in G$ such that $i \equiv g \equiv \lambda$ and $x=r_{i} g q_{\lambda}$; in fact $i=R_{x}, \lambda=L_{x}$.

Now take $x=r_{i} g q_{\lambda}, y=r_{j} h q_{\mu}$, with $i \equiv g \equiv \lambda, j \equiv h \equiv \mu$; then $x y=r_{k} l q_{\nu}$ with $k \equiv l \equiv \nu$; in the rest of this section we describe what we need to know in order to determine $k, l, \nu$ from $i, g, \lambda, j, h, \mu$.

2. The main idea is that we can describe the arbitrary product $x y$ if we know all the products of a certain type (hereafter called simple products). These simple products are analyzed in terms of the order relations on $I$ and $\Lambda$ that are induced by the $R$ and $\mathcal{L}$ preorders on $S$. The first type of simple product is actually the most complicated of all. It is analyzed in

LEMMA 1.2. Take $\lambda \in \Lambda, i \in I$, and write $q_{\lambda} r_{i}=r_{m} k q_{\nu}$, where $m \equiv \nu \equiv k \in$ $G$. Then $i \equiv i_{\alpha}$ implies $\nu \leqslant \lambda_{\alpha}, \lambda \equiv \lambda_{\alpha}$ implies $m \leqslant i_{\alpha}$.

Proof. Assume $i \equiv i_{\alpha}$, so that $r_{i} e_{\alpha}=r_{i}$. Then also $q_{\lambda} r_{i} e_{\alpha}=q_{\lambda} r_{i}$, which 
shows that $q_{\lambda} r_{i} \leqslant e_{\alpha}(\mathscr{L})$. Taking the $\mathscr{L}$-classes of both sides, we obtain $\nu \leqslant \lambda_{\alpha}$. The other part is dual.

When $i \in I, u \in I \cup G \cup \Lambda$, we write $i \prec u$ to mean $i \leqslant i_{\alpha} \equiv u$. We see that $i \prec u \equiv v$ implies $i \prec v ; j<i \prec u$ implies $j \prec u$; however, $\prec$ is not transitive. We can restate 1.2 as part of

Lemma 1.3. For each $i \in I, \lambda \in \Lambda$, there exist $i * \lambda \in I, \lambda * i \in \Lambda, \tau_{\lambda, i} \in G$ unique such that $i * \lambda \equiv \tau_{\lambda, i} \equiv \lambda * i$ and $q_{\lambda} r_{i}=r_{i * \lambda} \tau_{\lambda, i} q_{\lambda . i}$. Furthermore, $i * \lambda \prec \lambda, \lambda * i \prec i ; i \prec \lambda_{\alpha}$ implies $i * \lambda_{\alpha}=i, \tau_{\lambda_{\alpha}, i}=1, \lambda_{\alpha} * i=\lambda_{\beta} \equiv i$, and $\lambda \prec i_{\alpha}$ implies $i_{\alpha} * \lambda=i_{\gamma} \equiv \lambda, \tau_{\lambda, i_{\alpha}}=1 ; \lambda * i_{\alpha}=\lambda$.

Proof. If $i<\lambda_{\alpha}$, i.e. $i \leqslant i_{\alpha}$, then $r_{i} \leqslant e_{\alpha}(\Re)$ and, hence, $r_{i}=e_{\alpha} r_{i}$; then $q_{\lambda_{\alpha}} r_{i}=e_{\alpha} r_{i}=r_{i}=r_{i} 1 e_{\beta}$, where $e_{\beta} \equiv i$; then it follows from the uniqueness in 1.1 that $i * \lambda_{\alpha}=i, \tau_{\lambda_{\alpha}, i}=1, \lambda_{\alpha} * i=\lambda_{\beta}(\equiv i)$. The rest of the lemma follows by duality or by 1.2 .

3. The next type of simple product is analyzed in

Lemma 1.4. Let $g \in G, m \in I$ be such that $m \prec g$. Then there exist $g m \in I, g^{m} \in G$ unique such that $g m \equiv g^{m}$ and $g r_{m}=r_{g m} g^{m}$. Furthermore, $g m \prec g$; if $g=1\left(=e_{\alpha}\right)$ then $g m=m, g^{m}=1$; if $m=i_{\alpha} \equiv g$, then $g m=i_{\alpha}$, $g^{m}=g ;$ in general, $g m \equiv g^{m} \equiv m$.

Proof. Say $g \in G_{\alpha}$. Then $m \leqslant i_{\alpha}$, i.e. $r_{m} \leqslant e_{\alpha}(\mathcal{R})$, so that $r_{m}=e_{\alpha} r_{m}$. This implies $g r_{m} \mathcal{L} r_{m}$ : for $g$ has an inverse $h$ in $G_{\alpha}$, and $h g r_{m}=e_{\alpha} r_{m}=r_{m}$. On the other hand, $g r_{m}=e_{\alpha} g r_{m}$ and, hence, $g r_{m}<e_{\alpha}(\mathcal{R})$. If $r_{m} \equiv e_{\beta}$ then we have $g r_{m}=r_{n} k e_{\beta}=r_{n} k$ (with $n \equiv k \equiv e_{\beta}, k \in G$ ) and, hence, there exist (by 1.1) unique $g m=n \in I, g^{m}=k \in G$ such that $g m \equiv g^{m} \equiv m$ and $g r_{m}=r_{g m} g^{m}$; if, conversely, $g r_{m}=r_{n} k$ with $n \equiv k \in G$, then also $g r_{m}=r_{n} k e_{\gamma}$ for some $\gamma$ and the uniqueness in 1.1 shows that $n=g m, k=g^{m}$ and $\gamma=\beta$ (i.e. $\left.g^{m} \equiv m\right)$. This proves the first and last assertions; and since $g r_{m}<e_{\alpha}(\Re)$, we have $g m \leqslant i_{\alpha}$ and $g m \prec g$. Now take $g=e_{\alpha}$; then $g r_{m}=e_{\alpha} r_{m}=r_{m}=r_{m} 1$ (with $1=e_{\beta} \equiv m$ the identity element of $G_{\beta}$ ) and by the uniqueness in the above it follows that $g m=m, g^{m}=1$. (We also write this as: $1 m=m$, $1^{m}=1$, whenever defined.) If finally we take $m=i_{\alpha}$, then $g r_{m}=g e_{\alpha}=g=$ $r_{i_{\alpha}} g$ and, hence, $g m=i_{\alpha}, g^{m}=g$.

Dually, we have

Lemma 1.5. Let $g \in G, \nu \in \Lambda$ be such that $\nu \prec g$. Then there exist $\nu g \in \Lambda$, $g_{\nu} \in G$ unique such that $\nu g \equiv g$ and $q_{\nu} g=g_{\nu} q_{\nu g}$. Furthermore, $\nu g<g$; if $g=1$, then $\nu g=\nu, g_{\nu}=1$; if $\nu=\lambda_{\alpha} \equiv g$, then $g=\lambda_{\alpha}, g_{\nu}=g$; in general, $\nu g \equiv g_{\nu} \equiv \nu$.

The associativity properties of these new operations will be analyzed more thoroughly in $\$ 3$. We can already note that 1.4 defines an action of each $G_{\alpha}$ 
on the principal ideal $I_{\alpha}=\left\{m \in I ; m \leqslant i_{\alpha}\right\}$ of $I$. If $g, h \in G_{\alpha}, m<i_{\alpha}$, we can calculate $g\left(h r_{m}\right)=g\left(r_{h m} h^{m}\right)=r_{g(h m)} g^{h m} h^{m}$ (since $h m \prec h \equiv g$, so $h m$ $\prec g$ ), whereas $(g h) r_{m}=r_{(g h) m}(g h)^{m}$. Thus, $(g h) m=g(h m)$, which shows that the action of $G_{\alpha}$ on our principal ideal is indeed a group action. (The other action, of $I_{\alpha}$ on $G$, satisfies $(g h)^{m}=g^{h m} h^{m}$.) We can say more:

Lemma 1.6. When $m, n \in I, m \leqslant n, g \in G$, then $g m \leqslant g n$. Hence $G_{\alpha}$ acts on $I_{\alpha}=\left\{m \in I ; m \leqslant i_{\alpha}\right\}$ by automorphisms.

Proof. When $m \leqslant n$, then $r_{m} \leqslant r_{n}(\Re)$, i.e. $r_{m} \in r_{n} S$; therefore $g r_{m} \in$ $g r_{n} S, g r_{m} \leqslant g r_{n}(\Re)$ and $g m \leqslant g n$.

Dually, $G_{\alpha}$ acts on $\Lambda_{\alpha}=\left\{\nu \in \Lambda ; \nu \leqslant \lambda_{\alpha}\right\}$ by automorphisms.

4. The last type of simple products is

Lemma 1.7. When $m, i \in I, m \prec i$, there exist $i+m \in I, \sigma_{i, m} \in G$ unique such that $i+m \equiv \sigma_{i, m}$ and $r_{i} r_{m}=r_{i+m} \sigma_{i, m}$. Furthermore, $i+m \leqslant i$; if $i=i_{\alpha}$, then $i+m=m, \sigma_{i, m}=1\left(\in G_{\alpha}\right)$; if $m=i_{\alpha} \equiv i$, then $i+m=i, \sigma_{i, m}=1$; in general $i+m \equiv \sigma_{i, m} \equiv m$.

Proof. Say $i \equiv i_{\alpha}$. Then $m \leqslant i_{\alpha}$, so that $e_{\alpha} r_{m}=r_{m}$, and $r_{i} \mathcal{E} e_{\alpha}$; hence $r_{i} r_{m} \mathcal{E} e_{\alpha} r_{m}=r_{m}$. On the other hand, $r_{i} r_{m} \in r_{i} S$, so $r_{i} r_{m} \leqslant r_{i}(\Re)$. If $m \equiv e_{\beta}$, then we have $r_{i} r_{m}=r_{j} k e_{\beta}=r_{j} k$, with $j \in I, j \equiv e_{\beta} \equiv k \in G$, and $j, k$ are unique with these properties; as in the proof of 1.4 we see that they are also unique such that $r_{i} r_{m}=r_{j} k, j \equiv k, j \in I, k \in G$. We put $j=i+m, k=\sigma_{i, m}$ and have proved the first and last assertions; furthermore $r_{i} r_{m} \leqslant r_{i}(\Re)$ shows $i+m \leqslant i$. Now take $i=i_{\alpha}$, then $r_{i} r_{m}=e_{\alpha} r_{m}=r_{m}=r_{m} 1$ (where $1=e_{\beta} \in$ $\left.G_{\beta}\right)$ and so $i+m=m, \sigma_{i, m}=1$. Finally take $m=i_{\alpha}$; then $r_{i} r_{m}=r_{i} e_{\alpha}$ and so $i+m=i, \sigma_{i, m}=1\left(=e_{\alpha}\right)$.

Dually, we have

Lemma 1.8. When $\lambda, \nu \in \Lambda, \nu \prec \lambda$, there exist $\nu+\lambda \in \Lambda, v_{\nu, \lambda} \in G$ unique such that $\nu+\lambda \equiv v_{\nu, \lambda}$ and $q_{\nu} q_{\lambda}=v_{\nu, \lambda} q_{\nu+\lambda}$. Furthermore, $\nu+\lambda \leqslant \lambda$; if $\lambda=\lambda_{\alpha}$, then $\nu+\lambda=\nu, v_{\nu, \lambda}=1$; if $\nu=\lambda_{\alpha} \equiv \lambda$, then $\nu+\lambda=\lambda, v_{\nu, \lambda}=1$; in general, $\nu+\lambda \equiv v_{\nu, \lambda} \equiv \nu$.

Again the associativity properties of these new operations will be investigated later. We can already note that 1.7 is rather analogous to 1.4 ; we can make the analogy more evident if we think of the partial operation + on $I$ defined by 1.7 as defining for each $i \equiv i_{\alpha}$ a mapping of $I_{\alpha} \quad(=\{m \in I$; $\left.\left.m \leqslant i_{\alpha}\right\}\right)$ into the principal ideal $\{j \in I ; j \leqslant i\}$; namely, $m \mapsto i+m$. Thus, $i_{\alpha}$ yields the identity map on $I_{\alpha}$. In general, this mapping induced by $i$ is again an isomorphism:

Lemma 1.9. For each $j \in I, j \leqslant i \equiv i_{\alpha}$, there is a unique $m \leqslant i_{\alpha}$ such that $j=i+m$; if similarly $k=i+n$, then $n<m$ if and only if $k<j$. Thus 
$m \mapsto i+m$ is an isomorphism of $\{m \in I ; m \prec i\}$ upon $\{j \in I ; j \leqslant i\}$.

Proof. Since the $\mathcal{L}$-class $\lambda_{\alpha}$ of $r_{i}$ contains the idempotent $e_{\alpha}$, and the $\Re$-class of $r_{i}$ also contains an idempotent, $r_{i}$ has an inverse $r_{i}^{\prime}$ in the $\Re$-class $i_{\alpha}$ of $e_{\alpha}$. Now assume $j \leqslant i$. This implies $r_{j} \leqslant r_{i}(\Re)$, and, hence, $r_{j}=r_{i} s=$ $r_{i} r_{i}^{\prime} r_{i} s$ for some $s \in S$. Put $r_{i}^{\prime} r_{i} s=r_{m} h q_{\mu}$, with $m \equiv \mu \equiv h \in G$. Since $r_{i}^{\prime} r_{i} s \leqslant$ $r_{i}^{\prime}(\Re)$, we have $m \leqslant i_{\alpha}$. Hence $r_{j}=r_{i} r_{m} h q_{\mu}=r_{i+m} g h q_{\mu}$ with $i+m \equiv g \equiv m$; in particular, $g \equiv m \equiv h$, so that $g, h$ lie in the same group, and so $i+m \equiv$ $\mu \equiv g h \in G$. By $1.1, i+m$ is then the $R$-class of $r_{j}$, i.e. $j=i+m$. This proves the existence part of the first assertion; the uniqueness clearly will follow from the order properties. To prove these, we note that $n<m$ in $I$ implies $r_{n} \leqslant r_{m}(\Re), r_{i} r_{n} \leqslant r_{i} r_{m}(\Re)$ and, taking $\Re$-classes, $i+n \leqslant i+m$ (note that $n \leqslant m \leqslant i_{\alpha}$ so that $i+n$ is defined). For the converse, we use the inverse $r_{i}^{\prime}$ of $r_{i}$ as above, noting that $r_{i}^{\prime} r_{i}=e_{\alpha}$ : if $n, m \leqslant i_{\alpha}$ and $i+n \leqslant i+$ $m$, then $r_{i} r_{n} \leqslant r_{i} r_{m}(\Re)$ and, hence, $r_{n}=e_{\alpha} r_{n}=r_{i}^{\prime} r_{i} r_{n} \leqslant r_{i}^{\prime} r_{i} r_{m}=r_{m}(\Re)$, i.e. $n<m$.

COROLlaRY 1.10. When two $R$-classes of a regular semigroup lie in the same $\mathscr{D}$-class, they generate isomorphic principal ideals in $S / R$.

This can also be proved directly: by following the same argument as in the proof of Farès' theorem [4], one will see that in fact two $R$-classes of any semigroup which lie in the same regular $\mathscr{D}$-class will generate isomorphic principal ideals in $S / \Re$.

COROLlaRY 1.11. When $S$ is a (0-) bisimple regular semigroup, then $S / R$, $S / \mathcal{L}$ are (0-) uniform partially ordered sets.

The author was advised that this corollary was obtained by G. Hickel in 1967 (he does not seem to have published it). Similar results are well known for $E_{S}[1]$.

5. Lemma 1.3 and Lemmas 1.4, 1.7 and their duals, provide us with three factor sets $\sigma, \tau, v$ and with six actions or partial actions of each of $I, \Lambda$ on $G$, $I, \Lambda$, as well as two partial actions of $G$ on $I, \Lambda$. Together with the equivalence relation $\equiv$ on $I \cup G \cup \Lambda$ induced by $\mathscr{D}$, these constitute the structure data of the regular semigroup $S$. Our interest in the structure data stems from the fact that $S$ is completely determined (up to isomorphism) by its structure data. More precisely:

THEOREM 1. Let $S$ be a regular semigroup, and $G, I, \Lambda$ and the structure data of $S$ be as above. Let $T$ be the set of all triples $(i, g, \lambda) \in I \times G \times \Lambda$ such that $i \equiv g \equiv \lambda$. When $(i, g, \lambda),(j, h, \mu) \in T$, put

$$
(i, g, \lambda)(j, h, \mu)=\left(i+\mu, \sigma_{i, g m} g^{m} \tau_{\lambda_{j}} h_{\nu} v_{\nu h, \mu}, \nu h+\mu\right),
$$


where $m=j * \lambda, \nu=\lambda * j$. This multiplication is well defined on $T$ and makes $T$ a regular semigroup isomorphic to $S$.

Proof. Take $x=r_{i} g q_{\lambda}, y=r_{j} h q_{\mu} \in S$ (with $i, j \in I, g, h \in G, \lambda, \mu \in \Lambda$, $i \equiv g \equiv \lambda, j \equiv h \equiv \mu$ ). By 1.3 , we know that $q_{\lambda} r_{j}=r_{m} \tau_{\lambda, j} q_{\nu}$, where $m=$ $j * \lambda, \nu=\lambda * j, m \equiv \tau_{\lambda, j} \equiv \nu, m \prec \lambda, \nu \prec j$. Then $m \prec \lambda \equiv g$ shows $m \prec$ $g$, and by 1.4 we have $g r_{m}=r_{g m} g^{m}$, with $g m \equiv g^{m} \equiv m$; similarly $\nu \prec h$ and, by $1.5, q_{\nu} h=h_{\nu} q_{\nu h}$, with $h_{\nu} \equiv \nu h \equiv \nu$. We see that $g m \equiv g^{m} \equiv \tau_{\lambda, j} \equiv h_{\nu}$ $\equiv \nu h$. Furthermore, 1.3, 1.4 tell us that $g m \prec g$, $\nu h \prec h$; hence $g m \prec i$ (as $g \equiv i$ ) and $r_{i} r_{g m}=r_{i+g m} \sigma_{i, g m}$ by 1.7 ; dually, $\nu h \prec \mu$, and $q_{\nu h} q_{\mu}=v_{\nu h, \mu} q_{\nu h+\mu}$; 1.7, 1.8 also say that $i+g m \equiv \sigma_{i, g m} \equiv g m, v_{\nu h, \mu} \equiv \nu h+\mu \equiv \nu h$. Thus we obtain

$$
x y=r_{i} g q_{\lambda} r_{j} h q_{\mu}=r_{i+g m} \sigma_{i, g m} g^{m} \tau_{\lambda, j} h_{\nu} v_{\nu h, \mu} q_{v h+\mu},
$$

with $i+g m \in I, v h+\mu \in \Lambda$ and

$$
i+g m \equiv \sigma_{i, g m} \equiv g^{m} \equiv \tau_{\lambda, j} \equiv h_{\nu} \equiv v_{\nu h, \mu} \equiv v h+\mu ;
$$

in particular, all the group terms are equivalent and so their product lies in $G$ and is equivalent to $i+g m$ and $\nu h+\mu$. This argument also shows that the multiplication we put on $T$ in the theorem is well defined; furthermore, we now see that the mapping $r_{i} g q_{\lambda} \mapsto(i, g, \lambda)(i \equiv g \equiv \lambda)$ of $S$ into $T$, which by 1.1 is bijective, is an isomorphism.

Of course, Theorem 1 is a superficial result which must be completed by a characterization of structure data of regular semigroups: i.e. conditions on $\sigma$, $\tau, v, g^{m}, g m$, etc. that make it possible to define a multiplication as in Theorem 1 and insure that it is a regular semigroup multiplication; we saw that $g(h m)=(g h) m,(g h)^{m}=g^{h m} h^{m}$ are necessary conditions of that sort. One more condition can be obtained from the theorem, and involves only the two star operations; it expresses regularity:

Proposition 1.12. Let $S$ be a regular semigroup and $i \in I, \lambda \in \Lambda$. Then $i \cap \lambda$ is an $\mathcal{H}$-class containing an idempotent if and only if $i \equiv \lambda$ (三 $e_{\alpha}$, say), $i * \lambda=i_{\alpha}, \lambda * i=\lambda_{\alpha}$. Hence, for each $i \in I$ there is some $\lambda \in \Lambda$ (and, dually, for each $\lambda \in \Lambda$ there is some $i \in I$ ) such that these three relations hold.

Proof. First, $i \cap \lambda$ is an $\mathcal{H}$-class if and only if $i \equiv \lambda$, and we may as well assume from the start that this is the case. Then assume $i \cap \lambda$ contains an idempotent $e$; we can write $e=r_{i} g q_{\lambda}$, with $i \equiv \lambda \equiv g \in G$ (i.e. $g \in G_{\alpha}$ ). With $i * \lambda=m, \lambda * i=\nu$, the equality $e=e^{2}$ reads: $r_{i} g q_{\mu}=r_{i+g m} \cdots q_{\nu g+\mu}$. This implies $i+g m=i, \nu g+\mu=\mu$, which in turn implies, by 1.9 and its dual, $g m=i_{\alpha}\left(\right.$ as $i+i_{\alpha}=i$ by 1.7) and $\nu g=\lambda_{\alpha}$, and $m=i_{\alpha}, \nu=\lambda_{\alpha}$ by 1.6 and its dual.

Conversely, assume that $m=i * \lambda=i_{\alpha}, \nu=\lambda * i=\lambda_{\alpha}$; let $g=\tau_{\lambda, i}^{-1} \in G_{\alpha}$ 
(in particular, $i \equiv g \equiv \lambda$ ) and $e=r_{i} g q_{\lambda}$. By 1.4, 1.7 and their duals, $g^{m}=g$, $g m=m=i_{\alpha}, \sigma_{i, g m}=1, i+g m=i, g_{\nu}=g, \nu g=\nu=\lambda_{\alpha}, v_{\nu g, \lambda}=1$ and $\nu g+$ $\lambda=\lambda$. Therefore $e e=r_{i} g \tau_{\lambda, i} g q_{\lambda}=e$ by the choice of $g$.

6. As an application of these results, we show how the Rees-Suschkewitsch theorem can be derived from the results obtained so far. This is, of course, a rather picayune application, and we will be able to do much more once we have the Associativity Lemma and its consequences; but it is interesting that the progress of semigroup theory does not seem to have shortened its proof very much. Of course, in all proofs there surely must be a certain amount of elementary work at the beginning, to show that when $S$ is completely 0 -simple, then $\mathcal{H}$ is a congruence, and $S / \mathcal{H}$ is a rectangular 0 -band; but from there on one has a right to expect any new proof to be smooth and natural (i.e. even more so than the proof in [3]). For example, coextension theory does not yield such a proof, despite its obvious power. If the approach in [5] is followed, one must first take advantage of the fact that $\mathcal{H}$ is the congruence in this case, to show from the coherence theorem in [6] that one may build from one maximal subgroup, with all $\varphi$ and $\chi$ maps the identity; the approach in [7] is more natural for this, since in that approach it suffices to compute the Leech category $\mathscr{D}(S / \mathcal{F})$. This done, one obtains the multiplication under the form $(i, g, \lambda)(j, h, \mu)=\left(i, g \sigma_{i, \lambda: j, \mu} h, \mu\right)$ (for nonzero products) . . . and then one has to establish that the factor set $\sigma_{i, \lambda, j, \mu}$ depends only on $\lambda$ and $j$ (or can be chosen as such), an unpleasant task which surely is no improvement on the classical proof.

On the other hand, consider applying the above. First we note from $S / \mathcal{H}$ that $I$ and $\Lambda$ are discrete with zero adjoined (obviously 0 -uniform). We are to select $i_{0}, \lambda_{0}$ for the trivial $\mathscr{D}$-class and $i_{1}, \lambda_{1}$ for $S \backslash 0$. We see that $i \prec g \in$ $G_{1}$ implies $i=i_{1}$ or $i=i_{0}$; if $i \neq i_{0}$, this implies $g^{i}=g, g i=i$. Also, $m \prec i$ implies $m=i_{0}$ or $m=i_{1}$, in which case $i+m=i$ and $\sigma_{i, m}=1$. The dual properties hold in $\Lambda$. Therefore the multiplication in Theorem 1 specializes, in the case of nonzero elements, to $(i, g, \lambda)(j, h, \mu)=\left(i, g \tau_{\lambda, j} h, \mu\right)$ (in case $\tau_{\lambda, j} \equiv e_{1}$ ) or $=0$ (in case $\tau_{\lambda, j} \equiv e_{0}=0$ ). The theorem, however, does not (yet) tell us that all such multiplications are associative; but the regularity of the sandwich matrix $\tau_{\lambda, i}$ is immediate by 1.12 .

\section{The Associativity Lemma.}

1. We saw in $\S 1$ that the multiplication of a regular semigroup is completely determined by all the simple products (i.e. the values of $q_{\lambda} r_{i}, g r_{m}(m \prec g)$, $\left.q_{\nu} g(\nu \prec g), r_{i} r_{m}(m \prec i), q_{\nu} q_{\lambda}(\nu \prec \lambda)\right)$. Now we show that the associativity of these simple products also determines the associativity of the whole multiplication. The result is similar to Light's Test, but somewhat more complicated.

Evidently we cannot start from a regular semigroup, and so must first 
formalize the basic properties of products obtained in $\$ 1$. Specifically, we define a regular-like groupoid $S$ [in the sense of Ore] by the following four axioms.

Axiom (R) (Structure AND Relations). $S$ is a groupoid (= set with a binary operation) together with an equivalence relation $\equiv$ on $S$, subsets $S^{\prime}$, $R, S^{\prime \prime}$, relations $\prec \subseteq S^{\prime} \times S, \prec \subseteq S^{\prime \prime} \times S$, transitive relations $\leqslant$ on $S^{\prime}$ and $\leqslant$ on $S^{\prime \prime}$, such that

$\left(\mathrm{R}_{1}\right) u \prec x \equiv y$ implies $u \prec y$, for all $x, y \in S, u \in S^{\prime} \cup S^{\prime \prime}$;

$\left(\mathrm{R}_{2}\right) u \leqslant v \prec x$ implies $u \prec x$, for all $x \in S, u, v \in S^{\prime}, S^{\prime \prime}$.

[For example, $S$ could be a regular semigroup, with $R=G, S^{\prime}=\left\{r_{i} ; i \in I\right\}$, $S^{\prime \prime}=\left\{q_{\lambda} ; \lambda \in \Lambda\right\}, \equiv=\mathscr{D}, \prec$ as in $\S 1$ and $\leqslant$ defined by $r_{i}<r_{j} \Leftrightarrow i \leqslant j$, $q_{\lambda} \leqslant q_{\mu} \Leftrightarrow \lambda \leqslant \mu$. In general there is no inconvenience in denoting both relations by the same symbol because in what follows it will create no confusion; and similarly for $\leqslant$. Note that $\prec$ need not be transitive.]

Axiom (D) (Decomposition). For each $x \in S$, there exist $a^{\prime} \in S^{\prime}, r \in R$, $a^{\prime \prime} \in S^{\prime \prime}$ such that $x=a^{\prime}\left(r b^{\prime \prime}\right)$ and $a^{\prime} \equiv r \equiv b^{\prime \prime}$.

[In a regular semigroup, this follows from 1.1. Uniqueness is not necessary for what follows.]

AXIOM (P) (LOCATION OF PRODUCTS). The following conditions hold for all $a^{\prime}, b^{\prime} \in S^{\prime}, r, s \in R, a^{\prime \prime}, b^{\prime \prime} \in S^{\prime \prime}$ :

$\left(\mathrm{P}_{1}\right) r \equiv s$ implies $r s \in R$ and $r s \equiv r \equiv s$;

$\left(\mathrm{P}_{2}\right) a^{\prime \prime} b^{\prime}=c^{\prime}\left(u d^{\prime \prime}\right)$ for some $c^{\prime} \in S^{\prime}, u \in R, d^{\prime \prime} \in S^{\prime \prime}$ such that $c^{\prime} \equiv u \equiv$ $d^{\prime \prime}$ and $c^{\prime} \prec a^{\prime \prime}, d^{\prime \prime} \prec b^{\prime}$;

$\left(\mathrm{P}_{3}\right) a^{\prime} \prec r$ implies $r a^{\prime}=c^{\prime} u$ for some $c^{\prime} \in S^{\prime}, u \in R$ such that $c^{\prime} \equiv u \equiv$ $a^{\prime}$ and $c^{\prime} \prec r$;

$\left(\mathrm{P}_{3}^{\prime}\right) a^{\prime \prime} \prec r$ implies $a^{\prime \prime} r=u d^{\prime \prime}$ for some $u \in R, d^{\prime \prime} \in S^{\prime \prime}$ such that $u \equiv d^{\prime \prime}$ $\equiv a^{\prime \prime}$ and $d^{\prime \prime}<r$;

$\left(\mathrm{P}_{4}\right) b^{\prime} \prec a^{\prime}$ implies $a^{\prime} b^{\prime}=c^{\prime} u$ for some $c^{\prime} \in S^{\prime}, u \in R$ such that $c^{\prime} \equiv u \equiv$ $b^{\prime}$ and $c^{\prime} \leqslant a^{\prime}$;

( $\left.\mathrm{P}_{4}^{\prime}\right) a^{\prime \prime} \prec b^{\prime \prime}$ implies $a^{\prime \prime} b^{\prime \prime}=u d^{\prime \prime}$ for some $u \in R, d^{\prime \prime} \in S^{\prime \prime}$ such that $u \equiv d^{\prime \prime} \equiv a^{\prime \prime}$ and $d^{\prime \prime} \leqslant b^{\prime \prime}$.

[In a regular semigroup, $\left(\mathrm{P}_{1}\right)$ is trivial, and the other conditions follow form $1.3,1.4,1.5,1.7,1.8$, respectively.]

Axiom (E) (IDENTITY ELEMENTs). There exists $E \subseteq S^{\prime} \cap R \cap S^{\prime \prime}$ which spans $\equiv$ (i.e. for each $x \in S, x \equiv e$ for some $e \in E$ ), and for every $e \in E$ and all $a^{\prime} \in S^{\prime}, r \in R, a^{\prime \prime} \in S^{\prime \prime}$ :

$\left(\mathrm{E}_{1}\right) r \equiv e$ implies $e r=r=r e$;

$\left(\mathrm{E}_{2}\right) a^{\prime} \equiv e \equiv a^{\prime \prime}$ implies $a^{\prime} e=a^{\prime}, e a^{\prime \prime}=a^{\prime \prime}$;

$\left(\mathrm{E}_{3}\right) a^{\prime}<e$ implies $a^{\prime} \prec e, a^{\prime \prime} \leqslant e$ implies $a^{\prime \prime} \prec e$.

[In a regular semigroup, the set $E$ of all $e_{\alpha}$ has these properties.]

In particular, we see that regular semigroups are regular-like. We also note 
that the definition of regular-like groupoids is self-dual.

In what follows, we use conventions which allow us to locate every element of $S$ on sight. Namely, all primed (double-primed) letters denote elements of $S^{\prime}\left(S^{\prime \prime}\right) ; e$ denotes an element of $E, x, y, z$ denote arbitrary elements of $S$, and all other unprimed letters denote elements of $R$. Also, it is convenient to extend the four relations $\leqslant, \prec$ to $S$ as follows: $x<y\left(x \leqslant c^{\prime}\right)$ means that $x=a^{\prime}\left(r b^{\prime \prime}\right)$ for some $a^{\prime}, r, b^{\prime \prime}$ with $a^{\prime} \equiv r \equiv b^{\prime \prime}$ and $a^{\prime}<y\left(a^{\prime} \leqslant c^{\prime}\right) ; x^{\prime \prime}<y$ $\left(x^{\prime \prime} \leqslant d^{\prime \prime}\right)$ are defined similarly by $b^{\prime \prime} \prec y\left(b^{\prime \prime} \leqslant d^{\prime \prime}\right)$ (with $b^{\prime \prime}$ as above).

2. We can now state the Associativity Lemma.

TheOREM 2 (Associativity LemMA). A regular-like groupoid $S$ is a semigroup if and only if the associative law holds for all products of the following types:

(1) $a^{\prime} \cdot\left(r b^{\prime \prime}\right) \cdot x\left(a^{\prime} \equiv r \equiv b^{\prime \prime}\right)$

(1') $x \cdot\left(a^{\prime} r\right) \cdot b^{\prime \prime}\left(a^{\prime} \equiv r \equiv b^{\prime \prime}\right)$

(2) $x \cdot a^{\prime} \cdot\left(r b^{\prime \prime}\right)\left(a^{\prime} \equiv r \equiv b^{\prime \prime}\right)$;

$\left(a^{\prime} r\right) \cdot b^{\prime \prime} \cdot x\left(a^{\prime} \equiv r \equiv b^{\prime \prime}\right)$

(3) $r \cdot s \cdot t(r \equiv s \equiv t)$;

(4) $r \cdot s \cdot a^{\prime}\left(a^{\prime} \prec s \equiv r\right)$;

(5) $a^{\prime \prime} \cdot r \cdot b^{\prime}\left(a^{\prime \prime} \prec r, b^{\prime} \prec r\right)$;

(6) $r \cdot a^{\prime} \cdot b^{\prime}\left(b^{\prime} \prec a^{\prime} \prec r\right)$;

(6') $a^{\prime \prime} \cdot b^{\prime \prime} \cdot r\left(a^{\prime \prime} \prec b^{\prime \prime} \prec r\right)$;

(7) $a^{\prime} \cdot b^{\prime} \cdot c^{\prime}\left(c^{\prime} \prec b^{\prime} \prec a^{\prime}\right)$;

(7') $a^{\prime \prime} \cdot b^{\prime \prime} \cdot c^{\prime \prime}\left(a^{\prime \prime} \prec b^{\prime \prime} \prec c^{\prime \prime}\right)$;

(8) $a^{\prime \prime} \cdot b^{\prime} \cdot c^{\prime}\left(c^{\prime} \prec b^{\prime}\right)$;

(8) $a^{\prime \prime} \cdot b^{\prime \prime} \cdot c^{\prime}\left(a^{\prime \prime} \prec b^{\prime \prime}\right)$.

It follows from (1), (1'), (2), (2') that $a^{\prime}\left(r b^{\prime \prime}\right)=\left(a^{\prime} r\right) b^{\prime \prime}$ whenever $a^{\prime} \equiv r \equiv$ $b^{\prime \prime}$. In addition, the associative law also holds in the following cases:

$$
\begin{array}{llll}
a^{\prime} \cdot r \cdot x & \left(a^{\prime} \equiv r\right) ; & x \cdot r \cdot b^{\prime \prime} & \left(r \equiv b^{\prime \prime}\right) ; \\
a^{\prime} \cdot b^{\prime \prime} \cdot x & \left(a^{\prime} \equiv b^{\prime \prime}\right) ; & x \cdot a^{\prime} \cdot b^{\prime \prime} & \left(a^{\prime} \equiv b^{\prime \prime}\right) ; \\
r \cdot b^{\prime \prime} \cdot x & \left(r \equiv b^{\prime \prime}\right) ; & x \cdot a^{\prime} \cdot r & \left(a^{\prime} \equiv r\right) .
\end{array}
$$

This follows from (E): in the first case, for instance, we have $r=r e$, where $r \equiv e \in S^{\prime \prime}$, and so the associativity for $a^{\prime} \cdot r \cdot x$ (i.e. $\left.a^{\prime}(r x)=\left(a^{\prime} r\right) x\right)$ follows from (1). The other cases are similar.

Also note that the conditions in Theorem 2 are self-dual, so that duality can be used for the proof.

Before we begin the proof, note, finally, that we have to establish the associative law in $S$, which by (D) is a nine-variable identity

$$
\left(a^{\prime} r a^{\prime \prime}\right)\left(\left(b^{\prime} s b^{\prime \prime}\right)\left(c^{\prime} t c^{\prime \prime}\right)\right)=\left(\left(a^{\prime} r a^{\prime \prime}\right)\left(b^{\prime} s b^{\prime \prime}\right)\right)\left(c^{\prime} t c^{\prime \prime}\right),
$$

in terms of the conditions in the theorem, which, except for the first four, are three-variable identities that involve only the products covered by Axiom (P).

The first step of the proof is to show that the general product in our regular-like groupoid can be expressed in terms of simple products, just as we 
did for regular semigroups in $\$ 1$. [This is not a necessary part of the proceedings, since the results could be added to Axiom (P) instead of derived from the other conditions; they are easy to verify directly in case $S$ is a regular semigroup.] What we need is only

Lemma 2.1. Assume (1), (1'), (2), (2') hold and $a^{\prime} \equiv r \equiv a^{\prime \prime}, b^{\prime} \equiv s \equiv b^{\prime \prime}$. Then

(a) for all $x=c^{\prime} t c^{\prime \prime}$ (with $\left.c^{\prime} \equiv t \equiv c^{\prime \prime}\right), x a^{\prime}{ }^{\prime}<c^{\prime}, x a^{\prime} \stackrel{2}{\gtrless} a^{\prime}, a^{\prime \prime} x<a^{\prime \prime}$, $a^{\prime \prime} x$ " $\leqslant c^{\prime \prime}$;

(b) $\left(r a^{\prime \prime}\right)\left(b^{\prime} s\right) \stackrel{<\prime}{<}$ and ${ }^{\prime \prime}<b^{\prime}$;

(c) $b^{\prime} \prec r$ implies $\left(a^{\prime} r\right)\left(b^{\prime} s\right)=f^{\prime} t$ with $b^{\prime} \equiv t \equiv f^{\prime}<a^{\prime}$;

$\left(c^{\prime}\right) a^{\prime \prime} \prec s$ implies $\left(r a^{\prime \prime}\right)\left(s b^{\prime \prime}\right)=t f^{\prime \prime}$ with $a^{\prime \prime} \equiv t \equiv f^{\prime \prime} \leqslant b^{\prime \prime}$.

Proof. Let $x=c^{\prime} t c^{\prime \prime}$, with $c^{\prime} \equiv t \equiv c^{\prime \prime}$. Then

$$
\begin{array}{rlrl}
x a^{\prime} & =\left(\left(c^{\prime} t\right) c^{\prime \prime}\right) a^{\prime}=\left(c^{\prime} t\right)\left(c^{\prime \prime} a^{\prime}\right) & \text { by }\left(2^{\prime}\right)\left(\text { as } c^{\prime} \equiv t \equiv d^{\prime \prime}\right) \\
& =\left(c^{\prime} t\right)\left(f^{\prime}\left(u f^{\prime \prime}\right)\right) & & \text { with } f^{\prime} \equiv u \equiv f^{\prime \prime}, f^{\prime} \prec c^{\prime \prime}, f^{\prime \prime} \prec a^{\prime}, \text { by }\left(\mathrm{P}_{2}\right) \\
& =c^{\prime}\left(t\left(f^{\prime}\left(u f^{\prime \prime}\right)\right)\right) & & \text { by }(1)\left(\text { as } c^{\prime} \equiv t\right) \\
& =c^{\prime}\left(\left(t f^{\prime}\right)\left(u f^{\prime \prime}\right)\right) & & \text { by }(2)\left(\text { as } f^{\prime} \equiv u \equiv f^{\prime \prime}\right) \\
& =c^{\prime}\left(\left(g^{\prime} v\right)\left(u f^{\prime \prime}\right)\right) & & \text { with } g^{\prime} \equiv v \equiv f^{\prime}, g^{\prime} \prec t, \text { by }\left(\mathrm{P}_{3}\right) \\
& & & \left(\text { as } f^{\prime} \prec c^{\prime \prime} \equiv t, \text { so } f^{\prime} \prec t\right) \\
& =c^{\prime}\left(g^{\prime}\left(v\left(u f^{\prime \prime}\right)\right)\right) & & \text { by }(1)\left(\text { as } g^{\prime} \equiv v\right) \\
& =c^{\prime}\left(g^{\prime}\left((v u) f^{\prime \prime}\right)\right) & & \text { by }\left(1^{\prime}\right)\left(\text { as } u \equiv f^{\prime \prime}\right) \\
& =\left(c^{\prime} g^{\prime}\right)\left((v u) f^{\prime \prime}\right) & & \text { by }(2)\left(\text { as } v u \in R, v u \equiv u \equiv v \equiv g^{\prime} \equiv f^{\prime \prime}, \text { by }\left(\mathrm{P}_{1}\right)\right) \\
& =\left(h^{\prime} w\right)\left((v u) f^{\prime \prime}\right) & & \text { with } h^{\prime} \equiv w \equiv g^{\prime}, h^{\prime} \leqslant c^{\prime}, \text { by }\left(\mathrm{P}_{4}\right) \\
& & & \left(\text { as } g^{\prime} \prec t \equiv c^{\prime}, \text { so } g^{\prime} \prec c^{\prime}\right) \\
& =h^{\prime}\left(w\left((v u) f^{\prime \prime}\right)\right) & & \text { by }(1)\left(\text { as } h^{\prime} \equiv w\right) \\
& =h^{\prime}\left((w(v u)) f^{\prime \prime}\right) & & \text { by }\left(1^{\prime}\right)\left(\text { as } f^{\prime \prime} \equiv v u \in R\right),
\end{array}
$$

where $w(v u) \in R, w(v u) \equiv w \equiv v u$ by $\left(\mathrm{P}_{1}\right)\left(\right.$ as $\left.w \equiv g^{\prime} \equiv v \equiv v u\right)$ and, hence, $h^{\prime} \equiv w(v u) \equiv f^{\prime \prime}$. Then $h^{\prime} \leqslant c^{\prime}$ shows $x a^{\prime} \leqslant c^{\prime}, f^{\prime \prime} \prec a^{\prime}$ shows $x a^{\prime}{ }^{\prime \prime} \prec a^{\prime}$. This proves the first half of (a) and the second half follows by duality.

Next, we have

$$
\begin{aligned}
\left(r a^{\prime \prime}\right)\left(b^{\prime} s\right) & =\left(\left(r a^{\prime \prime}\right) b^{\prime}\right) s & & \text { by }(2)\left(\text { as } b^{\prime} \equiv s\right) \\
& =\left(\left(e r a^{\prime \prime}\right) b^{\prime}\right) s & & \text { with } e \equiv r \equiv a^{\prime \prime}, \text { by }(\mathrm{E}) \\
& =\left(f^{\prime} u g^{\prime \prime}\right) s & & \text { with } f^{\prime} \equiv u \equiv g^{\prime \prime}, f^{\prime}<e, g^{\prime \prime}<b^{\prime}, \text { by (a) } \\
& =\left(f^{\prime} u\right)\left(g^{\prime \prime} s\right) & & \text { by }\left(2^{\prime}\right)\left(\text { as } f^{\prime} \equiv u \equiv g^{\prime \prime}\right)
\end{aligned}
$$

further 


$$
\begin{aligned}
\left(r a^{\prime \prime}\right)\left(b^{\prime} s\right) & =\left(f^{\prime} u\right)\left(v f^{\prime \prime}\right) & & \text { with } v \equiv f^{\prime \prime} \equiv g^{\prime \prime}, f^{\prime \prime} \prec s, \text { by }\left(\mathrm{P}_{3}^{\prime}\right)\left(\text { as } g^{\prime \prime} \prec b^{\prime} \equiv s\right) \\
& =\left(\left(f^{\prime} u\right) v\right) f^{\prime \prime} & & \text { by }\left(1^{\prime}\right)\left(\text { as } v \equiv f^{\prime \prime}\right) \\
& =\left(f^{\prime}(u v)\right) f^{\prime \prime} & & \text { by }(1)\left(\text { as } f^{\prime} \equiv u\right),
\end{aligned}
$$

where $u \equiv g^{\prime \prime} \equiv v$ and so $u v \in R, u v \equiv u \equiv v \equiv f^{\prime} \equiv f^{\prime \prime}$ by $\left(\mathrm{P}_{1}\right)$. Since $f^{\prime} \prec$ $e \equiv a^{\prime \prime}$ by $\left(\mathrm{E}_{3}\right)$ and $f^{\prime \prime} \prec s \equiv b^{\prime}$, this proves (b).

Finally, assume $b^{\prime} \prec r$. Then

$$
\begin{aligned}
\left(a^{\prime} r\right)\left(b^{\prime} s\right) & =a^{\prime}\left(r\left(b^{\prime} s\right)\right) & & \text { by }(1)\left(\text { as } a^{\prime} \equiv r\right) \\
& =a^{\prime}\left(\left(r b^{\prime}\right) s\right) & & \text { by }(2)\left(\text { as } b^{\prime} \equiv s\right) \\
& =a^{\prime}\left(\left(c^{\prime} u\right) s\right) & & \text { with } c^{\prime} \equiv u \equiv b^{\prime}, c^{\prime} \prec r, \text { by }\left(\mathrm{P}_{3}\right) \\
& =a^{\prime}\left(c^{\prime}(u s)\right) & & \text { by }(1)\left(\text { as } c^{\prime} \equiv u\right) \\
& =\left(a^{\prime} c^{\prime}\right)(u s) & & \text { by }(2)\left(\text { as } c^{\prime} \equiv u \equiv s \equiv u s \in R \text { by }\left(\mathrm{P}_{1}\right)\right) \\
& =\left(f^{\prime} v\right)(u s) & & \text { with } f^{\prime} \equiv v \equiv c^{\prime}, f^{\prime} \leqslant a^{\prime} \text {, by }\left(\mathrm{P}_{4}\right)\left(\text { as } c^{\prime} \prec r \equiv a^{\prime}\right) \\
& =f^{\prime}(v(u s)) & & \text { by }(1)\left(\text { as } f^{\prime} \equiv v\right)
\end{aligned}
$$

where $v \equiv c^{\prime} \equiv u s \in R$ and, hence, $f^{\prime} \equiv v \equiv v(u s) \in R$ by $\left(\mathrm{P}_{1}\right)$. Since also $f^{\prime}<a^{\prime}$ and $f^{\prime} \equiv c^{\prime} \equiv b^{\prime}$, this proves (c), ( $\left.c^{\prime}\right)$ is dual of (c).

3. We now begin to sharpen the associativity properties that form our hypothesis.

Lemma 2.2. Conditions (1) to (4') imply associativity for the following products:

$(1+)\left(a^{\prime} r\right) \cdot\left(s b^{\prime \prime}\right) \cdot x$, with $a^{\prime} \equiv r \equiv s \equiv b^{\prime \prime}$,

$(2+) x \cdot\left(a^{\prime} r\right) \cdot\left(s b^{\prime \prime}\right)$, with $a^{\prime} \equiv r \equiv s \equiv b^{\prime \prime}$,

$(4+) r \cdot s \cdot x$, with $x<s \equiv r$.

Proof. We show $(4+)$ first. Assume $x<s \equiv r$, say $x=a^{\prime} t a^{\prime \prime}$, where $a^{\prime} \prec s$. Then:

$$
\begin{array}{rlrl}
r(s x) & =r\left(s\left(a^{\prime}\left(t a^{\prime \prime}\right)\right)\right)=r\left(\left(s a^{\prime}\right)\left(t a^{\prime \prime}\right)\right) & \text { by }(2)\left(\text { as } a^{\prime} \equiv t \equiv a^{\prime \prime}\right) \\
& =r\left(\left(b^{\prime} u\right)\left(t a^{\prime \prime}\right)\right) & & \text { with } b^{\prime} \equiv u \equiv a^{\prime}, b^{\prime} \prec s, \text { by }\left(\mathrm{P}_{3}\right) \\
& =r\left(b^{\prime}\left(u\left(t a^{\prime \prime}\right)\right)\right) & & \text { by }(1)\left(\text { as } b^{\prime} \equiv u\right) \\
& =r\left(b^{\prime}\left((u t) a^{\prime \prime}\right)\right) & & \text { by }\left(1^{\prime}\right)\left(\text { as } t \equiv a^{\prime \prime}\right) \\
& =\left(r b^{\prime}\right)\left((u t) a^{\prime \prime}\right) & & \text { by }\left(1^{\prime}\right)\left(\text { as } a^{\prime \prime} \equiv t \equiv u \equiv u t \in R \text { by }\left(\mathrm{P}_{1}\right)\right) \\
& =\left(c^{\prime} v\right)\left((u t) a^{\prime \prime}\right) & & \text { with } c^{\prime} \equiv v \equiv b^{\prime}, c^{\prime} \prec r, \text { by }\left(\mathrm{P}_{3}\right) \\
& & & \left(\text { as } b^{\prime} \prec s \equiv r\right) \\
& =c^{\prime}\left(v\left((u t) a^{\prime \prime}\right)\right) & & \text { by }(1)\left(\text { as } c^{\prime} \equiv v\right)
\end{array}
$$

further 


$$
\begin{aligned}
r(s x) & =c^{\prime}\left((v(u t)) a^{\prime \prime}\right) & & \text { by }\left(1^{\prime}\right)\left(\text { as } a^{\prime \prime} \equiv u t \in R\right) \\
& =\left(c^{\prime}(v(u t))\right) a^{\prime \prime} & & \text { by }(1)\left(\text { as } c^{\prime} \equiv v \equiv b^{\prime} \equiv u \equiv u t\right. \\
& & & \\
& =\left(c^{\prime}((v u) t)\right) a^{\prime \prime} & & \text { by }(3)\left(\text { as } v \equiv u \text { by }\left(\mathrm{P}_{1}\right)\right) \\
& =\left(\left(c^{\prime}(v u)\right) t\right) a^{\prime \prime} & & \text { by }(1)\left(\text { as } c^{\prime} \equiv v \equiv u \equiv v u \in R \text { by }\left(\mathrm{P}_{1}\right)\right) \\
& =\left(c^{\prime}(v u)\right)\left(t a^{\prime \prime}\right) & & \text { by }\left(1^{\prime}\right)\left(\text { as } t \equiv a^{\prime \prime}\right) \\
& =\left(\left(c^{\prime} v\right) u\right)\left(t a^{\prime \prime}\right) & & \text { by }(1)\left(\text { as } c^{\prime} \equiv v\right) \\
& =\left(\left(r b^{\prime}\right) u\right)\left(t a^{\prime \prime}\right) & & \left(\text { as } c^{\prime} v=r b^{\prime} \text { by definition }\right) \\
& =\left(r\left(b^{\prime} u\right)\right)\left(t a^{\prime \prime}\right) & & \text { by }(2)\left(\text { as } b^{\prime} \equiv u\right) \\
& =\left(r\left(s a^{\prime}\right)\right)\left(t a^{\prime \prime}\right) & & \left(\text { as } b^{\prime} u=s a^{\prime} \text { by definition }\right) \\
& =\left((r s) a^{\prime}\right)\left(t a^{\prime \prime}\right) & & \text { by (4) (as } \left.a^{\prime}<s \equiv r\right) \\
& =(r s)\left(a^{\prime}\left(t a^{\prime \prime}\right)\right)=(r s) x & & \text { by (2) and the definition of } x .
\end{aligned}
$$

This proves $(4+)$. [Dually, associativity holds for products $\left(4^{\prime}+\right) x \cdot r \cdot s$, with $x "<r \equiv s$.]

We now prove $(1+)$. Assuming $a^{\prime} \equiv r \equiv s \equiv b^{\prime \prime}$, we have

$$
\begin{aligned}
& \left.\left(a^{\prime} r\right)\left(\left(s b^{\prime \prime}\right) x\right)=a^{\prime}\left(r\left(\left(s b^{\prime \prime}\right) x\right)\right) \text { by (1) (as } a^{\prime} \equiv r\right) \\
& =a^{\prime}\left(r\left(s\left(b^{\prime \prime} x\right)\right)\right) \quad \text { by }\left(2^{\prime}\right)\left(\text { as } s \equiv b^{\prime \prime}\right) \\
& =a^{\prime}\left((r s)\left(b^{\prime \prime} x\right)\right) \quad \text { by }(4+)\left(\text { as } b^{\prime \prime} x^{\prime} \prec b^{\prime \prime} \equiv s \text { by } 2.1(\mathrm{a})\right. \\
& \text { and hence } b^{\prime \prime} x^{\prime}<s \equiv r \text { ) } \\
& =a^{\prime}\left(\left((r s) b^{\prime \prime}\right) x\right) \quad \text { by }\left(2^{\prime}\right)\left(\text { as } b^{\prime \prime} \equiv r \equiv s \equiv r s \in R \text { by }\left(\mathrm{P}_{1}\right)\right) \\
& =\left(a^{\prime}\left((r s) b^{\prime \prime}\right)\right) x \quad \text { by }(1)\left(\text { as } a^{\prime} \equiv r s \equiv b^{\prime \prime}\right) \\
& =\left(a^{\prime}\left(r\left(s b^{\prime \prime}\right)\right)\right) x=\left(\left(a^{\prime} r\right)\left(s b^{\prime \prime}\right)\right) x \quad \text { by }\left(1^{\prime}\right),(1)\left(\text { as } s \equiv b^{\prime \prime}, a^{\prime} \equiv r\right) \text {. }
\end{aligned}
$$

This proves $(1+)$. Observe that $(2+)$ is the dual of $(1+)$. $\square$

Note that we have begun to use conditions (3) to $\left(8^{\prime}\right)$. It does not seem possible to derive $(1+),(2+)$ from only (1), (2) and their duals.

Corollary 2.3. Conditions (1) to (4') also imply associativity in the following cases:

(9a) $\left(a^{\prime} b^{\prime}\right) \cdot\left(s c^{\prime \prime}\right) \cdot x$, with $c^{\prime \prime} \equiv s \equiv b^{\prime} \prec a^{\prime}$,

(9b) $\left(a^{\prime} r\right) \cdot\left(b^{\prime \prime} c^{\prime \prime}\right) \cdot x$, with $a^{\prime} \equiv r \equiv b^{\prime \prime} \prec c^{\prime \prime}$,

(9c) $\left(r b^{\prime}\right) \cdot\left(s c^{\prime \prime}\right) \cdot x$, with $c^{\prime \prime} \equiv s \equiv b^{\prime} \prec r$,

$\left(9 \mathrm{a}^{\prime}\right) x \cdot\left(a^{\prime} r\right) \cdot\left(b^{\prime \prime} c^{\prime \prime}\right)$, with $a^{\prime} \equiv r \equiv b^{\prime \prime} \prec c^{\prime \prime}$;

$\left(9 b^{\prime}\right) x \cdot\left(a^{\prime} b^{\prime}\right) \cdot\left(s c^{\prime \prime}\right)$, with $c^{\prime \prime} \equiv s \equiv b^{\prime} \prec a^{\prime}$,

(9d) $x \cdot\left(r b^{\prime}\right) \cdot\left(s c^{\prime \prime}\right)$, with $c^{\prime \prime} \equiv s \equiv b^{\prime} \prec r$. 
Proof. When $c^{\prime \prime} \equiv s \equiv b^{\prime} \prec a^{\prime}$, then by $\left(\mathrm{P}_{4}\right) a^{\prime} b^{\prime}=c^{\prime} u$ with $c^{\prime} \equiv u \equiv b^{\prime}$ $\equiv s \equiv c^{\prime \prime}$; hence $(9 \mathrm{a})$ follows from $(1+)$. The other formulae are established similarly, or follow by duality.

Further sharpening of (6), (7), (8) is given by the next lemma:

LEMMA 2.4. Conditions (1) to ( $\left(^{\prime}\right)$ imply associativity in the following cases:

(10) $r \cdot x \cdot a^{\prime}$, with $x x^{\prime}<r$ :

$\left(6^{*}\right) r \cdot a^{\prime} \cdot b^{\prime}$, with $b^{\prime} \prec a^{\prime}$;

(7*) $a^{\prime} \cdot b^{\prime} \cdot c^{\prime}$, with $c^{\prime} \prec b^{\prime}$

$\left(8^{\prime}+\right) a^{\prime \prime} \cdot b^{\prime \prime} \cdot c^{\prime} t$, with $a^{\prime \prime} \prec b^{\prime \prime}, c^{\prime} \equiv t$.

Proof. Assume $x^{\prime}<r$, i.e. $x=b^{\prime} s b^{\prime \prime}$ with $b^{\prime} \equiv s \equiv b^{\prime \prime}$ and $b^{\prime} \prec r$. Then

$$
\begin{array}{rlrl}
r\left(x a^{\prime}\right)= & r\left(\left(b^{\prime}\left(s b^{\prime \prime}\right)\right) a^{\prime}\right)=r\left(b^{\prime}\left(\left(s b^{\prime \prime}\right) a^{\prime}\right)\right) & \text { by }(1) \\
& =r\left(b^{\prime}\left(f^{\prime}\left(u f^{\prime \prime}\right)\right)\right) & & \text { where }\left(s b^{\prime \prime}\right) a^{\prime}=f^{\prime}\left(u f^{\prime \prime}\right) \\
& \text { with } f^{\prime} \equiv u \equiv f^{\prime \prime}, \\
& f^{\prime}<e \equiv s, \text { by } 2.1(\text { a }) \\
=r\left(\left(b^{\prime} f^{\prime}\right)\left(u f^{\prime \prime}\right)\right) & & \text { by }(2)\left(\text { as } f^{\prime} \equiv u \equiv f^{\prime \prime}\right) \\
=\left(r\left(b^{\prime} f^{\prime}\right)\right)\left(u f^{\prime \prime}\right) & & \text { by }\left(9 b^{\prime}\right)\left(\text { as } f^{\prime \prime} \equiv u \equiv f^{\prime} \prec e \equiv s \equiv b^{\prime}\right) \\
=\left(\left(r b^{\prime}\right) f^{\prime}\right)\left(u f^{\prime \prime}\right) & & \text { by }(6)\left(\text { as } f^{\prime} \prec b^{\prime}\right) \\
=\left(r b^{\prime}\right)\left(f^{\prime}\left(u f^{\prime \prime}\right)\right) & & \text { by }(2)\left(\text { as } f^{\prime} \equiv u \equiv f^{\prime \prime}\right) \\
=\left(r b^{\prime}\right)\left(\left(s b^{\prime \prime}\right) a^{\prime}\right) & & \text { as } f^{\prime}\left(u f^{\prime \prime}\right)=\left(s b^{\prime \prime}\right) a^{\prime} \\
=\left(\left(r b^{\prime}\right)\left(s b^{\prime \prime}\right)\right) a^{\prime} & & \text { by definition }(9 c)\left(\text { as } b^{\prime \prime} \equiv s \equiv b^{\prime} \prec r\right) \\
=\left(r\left(b^{\prime}\left(s b^{\prime \prime}\right)\right)\right) a^{\prime}=(r x) a^{\prime} & & \text { by }(2) .
\end{array}
$$

This proves (10). Note that, dually, associativity holds for

$$
a^{\prime \prime} \cdot x \cdot r \text {, with } x \stackrel{\text { " } r}{ } \text {. }
$$

Now assume $b^{\prime} \prec a^{\prime}$. Then

$$
\begin{array}{rlrl}
r\left(a^{\prime} b^{\prime}\right) & =(r e)\left(a^{\prime} b^{\prime}\right) & & \text { where } r \equiv e, \text { by }(\mathrm{E}) \\
& =r\left(e\left(a^{\prime} b^{\prime}\right)\right) & & \text { by }\left(2^{\prime}\right)\left(\text { as } r \equiv e \in S^{\prime \prime}\right) \\
& =r\left(\left(e a^{\prime}\right) b^{\prime}\right) & & \text { by }(8)\left(\text { as } e \in S^{\prime \prime}, b^{\prime} \prec a^{\prime}\right) \\
& =\left(r\left(e a^{\prime}\right)\right) b^{\prime} & & \text { by }(10)\left(\text { as } e a^{\prime} \cdot e e \equiv r, \text { by }\left(\mathrm{P}_{2}\right),\right. \\
& & \text { whence } \left.e a^{\prime}<r\right) \\
& \left.=\left((r e) a^{\prime}\right) b^{\prime}=\left(r a^{\prime}\right) b^{\prime}\right) & & \text { by }\left(2^{\prime}\right)\left(\text { as } r \equiv e \in S^{\prime \prime}\right) .
\end{array}
$$

This proves $\left(6^{*}\right)$.

Similarly, assume $c^{\prime} \prec b^{\prime}$. Then 


$$
\begin{aligned}
a^{\prime}\left(b^{\prime} c^{\prime}\right) & =\left(a^{\prime} e\right)\left(b^{\prime} c^{\prime}\right) & & \text { where } a^{\prime} \equiv e, \text { by }(\mathrm{E}) \\
& =a^{\prime}\left(e\left(b^{\prime} c^{\prime}\right)\right) & & \text { by }\left(2^{\prime}\right)\left(\text { as } a^{\prime} \equiv e \in S^{\prime \prime}\right) \\
& =a^{\prime}\left(\left(e b^{\prime}\right) c^{\prime}\right) & & \text { by }\left(6^{*}\right)\left(\text { as } e \in R, c^{\prime}<b^{\prime}\right) \\
& =a^{\prime}\left(\left(f^{\prime}\left(r f^{\prime \prime}\right)\right) c^{\prime}\right) & & \text { where } e b^{\prime}=f^{\prime} r f^{\prime \prime}, f^{\prime} \equiv r \equiv f^{\prime \prime}, \\
& & & f^{\prime} \prec e, f^{\prime \prime}<b^{\prime}, \text { by }\left(\mathrm{P}_{2}\right)\left(\text { as } e \in S^{\prime \prime}\right) \\
& =a^{\prime}\left(f^{\prime}\left(\left(r f^{\prime \prime}\right) c^{\prime}\right)\right) & & \text { by }(1)\left(\text { as } f^{\prime} \equiv r \equiv f^{\prime \prime}\right) \\
& =a^{\prime}\left(f^{\prime}\left(g^{\prime}\left(s g^{\prime \prime}\right)\right)\right) & & \text { where }\left(r f^{\prime \prime}\right) c^{\prime}=g^{\prime}\left(s g^{\prime \prime}\right) \text { with } g^{\prime} \equiv s \equiv e_{1} \equiv r, \text { by } 2.1(\text { a }) \\
& =a^{\prime}\left(\left(f^{\prime} g^{\prime}\right)\left(s g^{\prime \prime}\right)\right) & & \text { by }(1)\left(\text { as } g^{\prime} \equiv s \equiv g^{\prime \prime}\right) \\
& =\left(a^{\prime}\left(f^{\prime} g^{\prime}\right)\right)\left(s g^{\prime \prime}\right) & & \text { by } \left.\left(9 b^{\prime}\right)\left(\text { as } g^{\prime \prime} \equiv s \equiv g^{\prime} \prec e_{1} \equiv r \equiv f^{\prime}\right), \text { so that } g^{\prime} \prec f^{\prime}\right) \\
& =\left(\left(a^{\prime} f^{\prime}\right) g^{\prime}\right)\left(s g^{\prime \prime}\right) & & \text { by }(7)\left(\text { as } g^{\prime} \prec f^{\prime} \prec e \equiv a^{\prime}\right) \\
& =\left(a^{\prime} f^{\prime}\right)\left(g^{\prime}\left(s g^{\prime \prime}\right)\right) & & \text { by }(2)\left(\text { as } g^{\prime} \equiv s \equiv g^{\prime \prime}\right) \\
& =\left(a^{\prime} f^{\prime}\right)\left(\left(r f^{\prime \prime}\right) c^{\prime}\right) & & \left(\text { as } g^{\prime}\left(s g^{\prime \prime}\right)=\left(r f^{\prime \prime}\right) c^{\prime} \text { by definition }\right) \\
& =\left(\left(a^{\prime} f^{\prime}\right)\left(r f^{\prime \prime}\right)\right) c^{\prime} & & \text { by }(9 a)\left(\text { as } f^{\prime \prime} \equiv r \equiv f^{\prime} \prec a^{\prime}\right) \\
& =\left(a^{\prime}\left(f^{\prime}\left(r f^{\prime \prime}\right)\right)\right) c^{\prime} & & \text { by }(2)\left(\text { as } f^{\prime} \equiv r \equiv f^{\prime \prime}\right) \\
& =\left(a^{\prime}\left(e b^{\prime}\right)\right) c^{\prime} & & \left(\text { as } f^{\prime}\left(r f^{\prime \prime}\right)=e b^{\prime} \text { by definition }\right) \\
& =\left(\left(a^{\prime} e\right) b^{\prime}\right) c^{\prime} & & \text { by }(1)\left(\text { as } a^{\prime} \equiv e \in R\right) \\
& =\left(a^{\prime} b^{\prime}\right) c^{\prime}, & &
\end{aligned}
$$

which proves $\left(7^{*}\right)$.

Finally, assume $a^{\prime \prime} \prec b^{\prime \prime}, c^{\prime} \equiv t$. Then

$$
\begin{aligned}
a^{\prime \prime}\left(b^{\prime \prime}\left(c^{\prime} t\right)\right) & =a^{\prime \prime}\left(\left(b^{\prime \prime} c^{\prime}\right) t\right) & & \text { by }(2)\left(\text { as } c^{\prime} \equiv t\right) \\
& =\left(a^{\prime \prime}\left(b^{\prime \prime} c^{\prime}\right)\right) t & & \text { by }\left(10^{\prime}\right)\left(\text { as } b^{\prime \prime} c^{\prime \prime \prime} c^{\prime} \equiv t \text { by }\left(\mathrm{P}_{2}\right)\right) \\
& =\left(\left(a^{\prime \prime} b^{\prime \prime}\right) c^{\prime}\right) t & & \text { by }\left(8^{\prime}\right)\left(\text { as } a^{\prime \prime}<b^{\prime \prime}\right) \\
& =\left(a^{\prime \prime} b^{\prime \prime}\right)\left(c^{\prime} t\right) & & \text { by }(2)\left(\text { as } c^{\prime} \equiv t\right),
\end{aligned}
$$

which proves $\left(8^{\prime}+\right)$.

4. We now establish more associativity formulae which, like (10), have a comparatively high number of variables.

LEMMA 2.5. Conditions (1) to ( $\left.8^{\prime}\right)$ imply associativity in the following cases:

(11) $a^{\prime} \cdot b^{\prime} \cdot x$, with $x^{\prime}<b^{\prime}$

(12) $\left(r a^{\prime \prime}\right) \cdot b^{\prime} \cdot x$, with $r \equiv a^{\prime \prime}, x^{\prime}<b^{\prime}$;

(13) $\left(r a^{\prime \prime}\right) \cdot s \cdot x$, with $r \equiv a^{\prime \prime}<s, x^{\prime}<s$;

(13') $x \cdot r \cdot\left(a^{\prime} s\right)$, with $s \equiv a^{\prime} \prec r, x^{\prime}<r$; 
(14) $a^{\prime} \cdot\left(b^{\prime} r\right) \cdot\left(c^{\prime} s\right)$, with $b^{\prime} \equiv r, c^{\prime} \equiv s, c^{\prime} \prec b^{\prime}$;

$\left(14^{\prime}\right)\left(r a^{\prime \prime}\right) \cdot\left(s b^{\prime \prime}\right) \cdot c^{\prime \prime}$, with $r \equiv a^{\prime \prime}, s \equiv b^{\prime \prime}, a^{\prime \prime} \prec b^{\prime \prime}$;

(15) $\left(r a^{\prime \prime}\right) \cdot\left(s b^{\prime \prime}\right) \cdot\left(c^{\prime} t\right)$, with $r \equiv a^{\prime \prime}, s \equiv b^{\prime \prime}, c^{\prime} \equiv t, a^{\prime \prime} \prec b^{\prime \prime}$.

Proof. Assume $x^{\prime}<b^{\prime}$, say $x=c^{\prime} r c^{\prime \prime}$ with $c^{\prime} \equiv r \equiv c^{\prime \prime}$ and $c^{\prime} \prec b^{\prime}$. Then

$$
\begin{aligned}
a^{\prime}\left(b^{\prime} x\right) & =a^{\prime}\left(b^{\prime}\left(c^{\prime}\left(r c^{\prime \prime}\right)\right)\right)=a^{\prime}\left(\left(b^{\prime} c^{\prime}\right)\left(r c^{\prime \prime}\right)\right) & & \text { by }(2) \\
& =\left(a^{\prime}\left(b^{\prime} c^{\prime}\right)\right)\left(r c^{\prime \prime}\right) & & \text { by }\left(9 b^{\prime}\right)\left(\text { as } c^{\prime \prime} \equiv r \equiv c^{\prime} \prec b^{\prime}\right) \\
& =\left(\left(a^{\prime} b^{\prime}\right) c^{\prime}\right)\left(r c^{\prime \prime}\right) & & \text { by }\left(7^{*}\right)\left(\text { as } c^{\prime} \prec b^{\prime}\right) \\
& =\left(a^{\prime} b^{\prime}\right)\left(c^{\prime}\left(r c^{\prime \prime}\right)\right)=\left(a^{\prime} b^{\prime}\right) x & & \text { by }(2) .
\end{aligned}
$$

This proves (11).

Now assume $r \equiv a^{\prime \prime}$ and $x^{\prime}<b^{\prime}$, say $x=c^{\prime} s c^{\prime \prime}$ with $c^{\prime} \equiv s \equiv c^{\prime \prime}$ and $c^{\prime} \prec b^{\prime}$. Then

$$
\begin{aligned}
\left(r a^{\prime \prime}\right)\left(b^{\prime} x\right) & =\left(r a^{\prime \prime}\right)\left(b^{\prime}\left(c^{\prime}\left(s c^{\prime \prime}\right)\right)\right)=\left(r a^{\prime \prime}\right)\left(\left(b^{\prime} c^{\prime}\right)\left(s c^{\prime \prime}\right)\right) \quad \text { by }(2) \\
& =\left(\left(r a^{\prime \prime}\right)\left(b^{\prime} c^{\prime}\right)\right)\left(s c^{\prime \prime}\right) \quad \text { by }\left(9 b^{\prime}\right)\left(\text { as } c^{\prime \prime} \equiv s \equiv c^{\prime} \prec b^{\prime}\right) \\
& =\left(r\left(a^{\prime \prime}\left(b^{\prime} c^{\prime}\right)\right)\right)\left(s c^{\prime \prime}\right) \quad \text { by }\left(2^{\prime}\right)\left(\text { as } r \equiv a^{\prime \prime}\right) \\
& =\left(r\left(\left(a^{\prime \prime} b^{\prime}\right) c^{\prime}\right)\right)\left(s c^{\prime \prime}\right) \quad \text { by }(8)\left(\text { as } c^{\prime}<b^{\prime}\right) \\
& =\left(\left(r\left(a^{\prime \prime} b^{\prime}\right)\right) c^{\prime}\right)\left(s c^{\prime \prime}\right) \quad \text { by }(10)\left(\text { as } a^{\prime \prime} b^{\prime \prime}<a^{\prime \prime} \equiv r \text { by }\left(\mathrm{P}_{2}\right)\right) \\
& =\left(r\left(a^{\prime \prime} b^{\prime}\right)\right)\left(c^{\prime}\left(s c^{\prime \prime}\right)\right) \quad \text { by }(2)\left(\text { as } c^{\prime} \equiv s \equiv c^{\prime \prime}\right) \\
& =\left(\left(r a^{\prime \prime}\right) b^{\prime}\right)\left(c^{\prime}\left(s c^{\prime \prime}\right)\right)=\left(\left(r a^{\prime \prime}\right) b^{\prime}\right) x \quad \text { by }\left(2^{\prime}\right)\left(\text { as } r \equiv a^{\prime \prime}\right)
\end{aligned}
$$

This proves (12).

Now assume $r \equiv a^{\prime \prime} \prec s$, and $x^{\prime} \prec s$, say $x=c^{\prime} t c^{\prime \prime}$ with $c^{\prime} \equiv t \equiv c^{\prime \prime}$ and $c^{\prime} \prec s$. Then

$$
\begin{array}{rlrl}
\left(r a^{\prime \prime}\right)(s x) & =\left(r a^{\prime \prime}\right)\left(s\left(c^{\prime}\left(t c^{\prime \prime}\right)\right)\right)=\left(r a^{\prime \prime}\right)\left(\left(s c^{\prime}\right)\left(t c^{\prime \prime}\right)\right) & \text { by }(2) \\
& =\left(\left(r a^{\prime \prime}\right)\left(s c^{\prime}\right)\right)\left(t c^{\prime \prime}\right) & & \text { by }(9 \mathrm{~d})\left(\text { as } c^{\prime \prime} \equiv t \equiv c^{\prime} \prec s\right) \\
& =\left(r\left(a^{\prime \prime}\left(s c^{\prime}\right)\right)\right)\left(t c^{\prime \prime}\right) & & \text { by }\left(2^{\prime}\right)\left(\text { as } r \equiv a^{\prime \prime}\right) \\
& =\left(r\left(\left(a^{\prime \prime} s\right) c^{\prime}\right)\right)\left(t c^{\prime \prime}\right) & & \text { by }(5)\left(\text { as } a^{\prime \prime} \prec s, c^{\prime} \prec s\right) \\
& =\left(\left(r\left(a^{\prime \prime} s\right)\right) c^{\prime}\right)\left(t c^{\prime \prime}\right) & & \text { by }(10)\left(\text { as } a^{\prime \prime} s^{\prime}<a^{\prime \prime} \equiv r \text { by } 2.1(\mathrm{a})\right) \\
& =\left(\left(\left(r a^{\prime \prime}\right) s\right) c^{\prime}\right)\left(t c^{\prime \prime}\right) & & \text { by }\left(2^{\prime}\right)\left(\text { as } r \equiv a^{\prime \prime}\right) \\
& =\left(\left(r a^{\prime \prime}\right) s\right)\left(c^{\prime}\left(t c^{\prime \prime}\right)\right) & & \text { by }(2)\left(\text { as } c^{\prime} \equiv t \equiv c^{\prime \prime}\right) \\
& =\left(\left(r a^{\prime \prime}\right) s\right) x, & &
\end{array}
$$

which proves (13). Then (13') follows by duality.

Next, assume $s \equiv c^{\prime} \prec b^{\prime} \equiv r$. Then 


$$
\begin{aligned}
& \left(a^{\prime}\left(b^{\prime} r\right)\right)\left(c^{\prime} s\right)=\left(\left(a^{\prime} b^{\prime}\right) r\right)\left(c^{\prime} s\right) \quad \text { by }(2)\left(\text { as } b^{\prime} \equiv r\right) \\
& =\left(a^{\prime} b^{\prime}\right)\left(r\left(c^{\prime} s\right)\right) \text { by }\left(13^{\prime}\right)\left(\text { as } s \equiv c^{\prime} \prec r\right. \text {, and } \\
& \left.a^{\prime} b^{\prime} "<b^{\prime} \equiv r \text { by } 2.1(\mathrm{a})\right) \\
& =\left(a^{\prime} b^{\prime}\right)\left(d^{\prime} t\right) \quad \text { where } r\left(c^{\prime} s\right)=(e r)\left(c^{\prime} s\right) \quad(\text { with } r \equiv e) \\
& =d^{\prime} t \text { with } t \equiv d^{\prime} \leqslant e \text {, by } 2.1 \text { (c) } \\
& \text { (hence also } d^{\prime} \prec e \equiv r \equiv b^{\prime} \text { by }\left(\mathrm{E}_{3}\right) \text { ) } \\
& \left.=\left(\left(a^{\prime} b^{\prime}\right) d^{\prime}\right) t \quad \text { by (2) (as } d^{\prime} \equiv t\right) \\
& =\left(a^{\prime}\left(b^{\prime} d^{\prime}\right)\right) t \quad \text { by }\left(7^{*}\right)\left(\text { as } d^{\prime} \prec b^{\prime}\right) \\
& =\left(a^{\prime}\left(f^{\prime} v\right)\right) t \quad \text { where } b^{\prime} d^{\prime}=f^{\prime} v, f^{\prime} \equiv v \equiv d^{\prime} \text {, by }\left(\mathrm{P}_{4}\right) \\
& \text { (as } d^{\prime} \prec b^{\prime} \text { ) } \\
& =a^{\prime}\left(\left(f^{\prime} v\right) t\right) \quad \text { by }(2+)\left(\text { as } f^{\prime} \equiv v \equiv d^{\prime} \equiv t\right) \\
& =a^{\prime}\left(\left(b^{\prime} d^{\prime}\right) t\right) \quad\left(\text { as } f^{\prime} v=b^{\prime} d^{\prime}\right. \text { by definition) } \\
& =a^{\prime}\left(b^{\prime}\left(d^{\prime} t\right)\right) \quad \text { by (2) }\left(\text { as } d^{\prime} \equiv t\right) \\
& =a^{\prime}\left(b^{\prime}\left(r\left(c^{\prime} s\right)\right)\right) \quad\left(\text { as } d^{\prime} t=r\left(c^{\prime} s\right) \text { by definition }\right) \\
& \left.=a^{\prime}\left(\left(b^{\prime} r\right)\left(c^{\prime} s\right)\right) \quad \text { by (1) (as } b^{\prime} \equiv r\right) \text {. }
\end{aligned}
$$

This proves (14). Then (14') follows by duality.

Finally, assume $r \equiv a^{\prime \prime} \prec b^{\prime \prime} \equiv s, c^{\prime} \equiv t$. Then

$$
\begin{aligned}
& \left(r a^{\prime \prime}\right)\left(\left(s b^{\prime \prime}\right)\left(c^{\prime} t\right)\right)=\left(r a^{\prime \prime}\right)\left(s\left(b^{\prime \prime}\left(c^{\prime} t\right)\right)\right) \text { by }\left(2^{\prime}\right)\left(\text { as } s \equiv b^{\prime \prime}\right) \\
& =\left(\left(r a^{\prime \prime}\right) s\right)\left(b^{\prime \prime}\left(c^{\prime} t\right)\right) \quad \text { by (13) (as } r \equiv a^{\prime \prime} \prec s \text { and } \\
& \left.b^{\prime \prime}\left(c^{\prime} t\right)<b^{\prime \prime} \equiv s \text { by } 2.1(\mathrm{a})\right) \\
& =\left(u f^{\prime \prime}\right)\left(b^{\prime \prime}\left(c^{\prime} t\right)\right) \quad \text { where }\left(r a^{\prime \prime}\right) s=\left(r a^{\prime \prime}\right)(s e)(\text { with } s \equiv e) \\
& =u f^{\prime \prime} \text { with } u \equiv f^{\prime \prime} \leqslant e \text {, by } 2.1\left(\mathrm{c}^{\prime}\right) \\
& \text { (as } a^{\prime \prime} \prec s \text { ) } \\
& =u\left(f^{\prime \prime}\left(b^{\prime \prime}\left(c^{\prime} t\right)\right)\right) \quad \text { by }\left(2^{\prime}\right)\left(\text { as } u \equiv f^{\prime \prime}\right) \\
& =u\left(\left(f^{\prime \prime} b^{\prime \prime}\right)\left(c^{\prime} t\right)\right) \quad \text { by }\left(8^{\prime}+\right)\left(\text { as } c^{\prime} \equiv t \text { and } f^{\prime \prime} \prec e \equiv s\right. \\
& \left.\equiv b^{\prime \prime} \text { by }\left(\mathrm{E}_{3}\right)\right) \\
& =\left(u\left(f^{\prime \prime} b^{\prime \prime}\right)\right)\left(c^{\prime} t\right) \quad \text { by }(9 \mathrm{~b})\left(\text { as } u \equiv f^{\prime \prime} \prec b^{\prime \prime}\right) \\
& =\left(\left(u f^{\prime \prime}\right) b^{\prime \prime}\right)\left(c^{\prime} t\right) \quad \text { by }\left(2^{\prime}\right)\left(\text { as } u \equiv f^{\prime \prime}\right) \\
& =\left(\left(\left(r a^{\prime \prime}\right) s\right) b^{\prime \prime}\right)\left(c^{\prime} t\right) \quad\left(\text { as } u f^{\prime \prime}=\left(r a^{\prime \prime}\right) s \text { by definition }\right) \\
& =\left(\left(r a^{\prime \prime}\right)\left(s b^{\prime \prime}\right)\right)\left(c^{\prime} t\right) \quad \text { by }\left(1^{\prime}\right) \text {. }
\end{aligned}
$$

This proves (15). 
The last lemma brings us four more formulae:

LEMMA 2.6. When (1) to $\left(8^{\prime}\right)$ hola, so does the associative law for the following products:

(16) $a^{\prime} \cdot x \cdot\left(b^{\prime} r\right)$, with $b^{\prime} \equiv r$;

$\left(16^{\prime}\right)\left(r a^{\prime \prime}\right) \cdot x \cdot b^{\prime \prime}$, with $r \equiv a^{\prime \prime}$;

(17) $\left(r a^{\prime \prime}\right) \cdot x \cdot\left(b^{\prime} s\right)$, with $r \equiv a^{\prime \prime}, b^{\prime} \equiv s$;

(18) $a^{\prime} \cdot x \cdot b^{\prime \prime}$.

Proof. We put $x=c^{\prime} t c^{\prime \prime}$ throughout, with $c^{\prime} \equiv t \equiv c^{\prime \prime}$. If we assume $b^{\prime} \equiv r$, then

$$
\begin{aligned}
& a^{\prime}\left(x\left(b^{\prime} r\right)\right)=a^{\prime}\left(\left(c^{\prime}\left(t c^{\prime \prime}\right)\right)\left(b^{\prime} r\right)\right)=a^{\prime}\left(c^{\prime}\left(\left(t c^{\prime \prime}\right)\left(b^{\prime} r\right)\right)\right) \text { by (1) } \\
& =\left(a^{\prime} c^{\prime}\right)\left(\left(t c^{\prime \prime}\right)\left(b^{\prime} r\right)\right) \quad \text { by }(11)\left(\text { as }\left(t c^{\prime \prime}\right)\left(b^{\prime} r\right)^{\prime} \prec c^{\prime \prime} \equiv c^{\prime}\right. \text {, } \\
& =\left(f^{\prime}\left(u f^{\prime \prime}\right)\right)\left(\left(t c^{\prime \prime}\right)\left(b^{\prime} r\right)\right) \quad \text { where } a^{\prime} c^{\prime}=f^{\prime} u f^{\prime \prime} \text { with } f^{\prime} \equiv u \equiv f^{\prime \prime} \\
& \text { and } f^{\prime \prime} \prec c^{\prime} \text {, by } 2.1(\mathrm{a}) \\
& =f^{\prime}\left(\left(u f^{\prime \prime}\right)\left(\left(t c^{\prime \prime}\right)\left(b^{\prime} r\right)\right)\right) \quad \text { by }(1)\left(\text { as } f^{\prime} \equiv u \equiv f^{\prime \prime}\right) \\
& =f^{\prime}\left(\left(\left(u f^{\prime \prime}\right)\left(t c^{\prime \prime}\right)\right)\left(b^{\prime} r\right)\right) \quad \text { by }(15) \text { (as } u \equiv f^{\prime \prime} \prec c^{\prime} \equiv t \equiv c^{\prime \prime} \text {, } \\
& =f^{\prime}\left(\left(v g^{\prime \prime}\right)\left(b^{\prime} r\right)\right) \quad \text { where }\left(u f^{\prime \prime}\right)\left(t c^{\prime \prime}\right)=v g^{\prime \prime} \text { with } \\
& v \equiv g^{\prime \prime} \equiv f^{\prime \prime} \text {, by } 2.1\left(\mathrm{c}^{\prime}\right) \\
& \text { (as } u \equiv f^{\prime \prime} \prec c^{\prime} \equiv t \equiv \mathrm{c}^{\prime \prime} \text { ) } \\
& =\left(f^{\prime}\left(v g^{\prime \prime}\right)\right)\left(b^{\prime} r\right) \quad \text { by }(1)\left(\text { as } f^{\prime} \equiv f^{\prime \prime} \equiv v \equiv g^{\prime \prime}\right) \\
& =\left(f^{\prime}\left(\left(u f^{\prime \prime}\right)\left(t c^{\prime \prime}\right)\right)\right)\left(b^{\prime} r\right) \quad\left(\text { as } v g^{\prime \prime}=\left(u f^{\prime \prime}\right)\left(t c^{\prime \prime}\right)\right) \\
& \left.=\left(\left(f^{\prime}\left(u f^{\prime \prime}\right)\right)\left(t c^{\prime \prime}\right)\right)\left(b^{\prime} r\right) \quad \text { by (1) (as } f^{\prime} \equiv u \equiv f^{\prime \prime}\right) \\
& =\left(\left(a^{\prime} c^{\prime}\right)\left(t c^{\prime \prime}\right)\right)\left(b^{\prime} r\right) \quad\left(\text { as } f^{\prime} u f^{\prime \prime}=a^{\prime} c^{\prime}\right. \text { by definition) } \\
& =\left(a^{\prime}\left(c^{\prime}\left(t c^{\prime \prime}\right)\right)\right)\left(b^{\prime} r\right)=\left(a^{\prime} x\right)\left(b^{\prime} r\right) \quad \text { by (2) }\left(\text { as } c^{\prime} \equiv t \equiv c^{\prime \prime}\right)
\end{aligned}
$$

which proves $(16)$. Then $\left(16^{\prime}\right)$ follows by duality.

Now assume $r \equiv a^{\prime \prime}, b^{\prime} \equiv s$. Then

$$
\begin{array}{rlr}
\left(r a^{\prime \prime}\right)\left(x\left(b^{\prime} s\right)\right) & =\left(r a^{\prime \prime}\right)\left(\left(c^{\prime}\left(t c^{\prime \prime}\right)\right)\left(b^{\prime} s\right)\right) \\
& =\left(r a^{\prime \prime}\right)\left(c^{\prime}\left(\left(t c^{\prime \prime}\right)\left(b^{\prime} s\right)\right)\right) & \text { by }(1) \\
& =\left(\left(r a^{\prime \prime}\right) c^{\prime}\right)\left(\left(t c^{\prime \prime}\right)\left(b^{\prime} s\right)\right) & \text { by }(1)\left(\text { as } r \equiv a^{\prime \prime},\right. \text { and } \\
& & \left.\left(t c^{\prime \prime}\right)\left(b^{\prime} s\right)<c^{\prime \prime} \equiv c^{\prime}, \text { by } 2.1(b)\right)
\end{array}
$$

further 


$$
\begin{aligned}
& \left(r a^{\prime \prime}\right)\left(x\left(b^{\prime} s\right)\right)=\left(f^{\prime}\left(u f^{\prime \prime}\right)\right)\left(\left(t c^{\prime \prime}\right)\left(b^{\prime} s\right)\right) \quad \text { where }\left(r a^{\prime \prime}\right) c^{\prime} \quad\left[=\left(r a^{\prime \prime}\right)\left(c^{\prime} e\right)\right. \text {, } \\
& \text { where } c^{\prime} \equiv e \text { ] }=f^{\prime} u f^{\prime \prime} \\
& \text { with } f^{\prime} \equiv u \equiv f^{\prime \prime} \\
& \text { and } f^{\prime \prime} \prec c^{\prime} \text {, by } 2.1(\mathrm{~b}) \\
& =\left(\left(f^{\prime}\left(u f^{\prime \prime}\right)\right)\left(t c^{\prime \prime}\right)\right)\left(b^{\prime} s\right) \quad \text { as in the proof of (16), above } \\
& =\left(\left(\left(r a^{\prime \prime}\right) c^{\prime}\right)\left(t c^{\prime \prime}\right)\right)\left(b^{\prime} s\right) \quad\left(\text { as }\left(r a^{\prime \prime}\right) c^{\prime}=f^{\prime} u f^{\prime \prime}\right) \\
& =\left(\left(r a^{\prime \prime}\right)\left(c^{\prime}\left(t c^{\prime \prime}\right)\right)\right)\left(b^{\prime} s\right) \quad \text { by }(2)\left(\text { as } c^{\prime} \equiv t \equiv c^{\prime \prime}\right) \\
& =\left(\left(r a^{\prime \prime}\right) x\right)\left(b^{\prime} s\right) \text {, }
\end{aligned}
$$

which proves (17).

Finally,

$$
\begin{aligned}
& a^{\prime}\left(x b^{\prime \prime}\right)=a^{\prime}\left(\left(c^{\prime}\left(t c^{\prime \prime}\right)\right) b^{\prime \prime}\right)=a^{\prime}\left(c^{\prime}\left(\left(t c^{\prime \prime}\right) b^{\prime \prime}\right)\right) \text { by (1) } \\
& =a^{\prime}\left(c^{\prime}\left(t\left(c^{\prime \prime} b^{\prime \prime}\right)\right)\right) \quad \text { by }\left(2^{\prime}\right)\left(\text { as } t \equiv c^{\prime \prime}\right) \\
& =a^{\prime}\left(c^{\prime}\left(t\left(c^{\prime \prime}\left(e b^{\prime \prime}\right)\right)\right)\right) \quad \text { with } e \equiv b^{\prime \prime} \text {, by (E) } \\
& =a^{\prime}\left(c^{\prime}\left(t\left(\left(c^{\prime \prime} e\right) b^{\prime \prime}\right)\right)\right) \quad \text { by }\left(1^{\prime}\right)\left(\text { as } b^{\prime \prime} \equiv e \in R\right) \\
& =a^{\prime}\left(c^{\prime}\left(t\left(\left(f^{\prime}\left(u f^{\prime \prime}\right)\right) b^{\prime \prime}\right)\right)\right) \quad \text { where } c^{\prime \prime} e=f^{\prime} u f^{\prime \prime} \text { with } \\
& f^{\prime} \equiv u \equiv f^{\prime \prime}, \\
& f^{\prime} \prec c^{\prime \prime}, f^{\prime \prime} \prec e, \\
& \text { by }\left(\mathrm{P}_{2}\right)\left(\text { as } e \in S^{\prime}\right) \\
& =a^{\prime}\left(c^{\prime}\left(t\left(f^{\prime}\left(\left(u f^{\prime \prime}\right) b^{\prime \prime}\right)\right)\right)\right) \quad \text { by }(1)\left(\text { as } f^{\prime} \equiv u \equiv f^{\prime \prime}\right) \\
& =a^{\prime}\left(c^{\prime}\left(t\left(f^{\prime}\left(v g^{\prime \prime}\right)\right)\right)\right) \quad \text { where }\left(u f^{\prime \prime}\right) b^{\prime \prime}=v g^{\prime \prime} \text { with } \\
& v \equiv g^{\prime \prime} \equiv f^{\prime \prime}, \text { by } 2.1\left(c^{\prime}\right) \\
& \text { (as } \left.f^{\prime \prime} \prec e \equiv b^{\prime \prime}\right) \\
& =a^{\prime}\left(c^{\prime}\left(\left(t f^{\prime}\right)\left(v g^{\prime \prime}\right)\right)\right) \quad \text { by }(2)\left(\text { as } f^{\prime} \equiv f^{\prime \prime} \equiv v \equiv g^{\prime \prime}\right) \\
& =a^{\prime}\left(c^{\prime}\left(\left(g^{\prime} w\right)\left(v g^{\prime \prime}\right)\right)\right) \quad \text { where } t f^{\prime}=\left(e_{1} t\right) f^{\prime}\left(\text { with } t \equiv e_{1}\right) \\
& =g^{\prime} w \text {, with } w \equiv g^{\prime} \equiv f^{\prime} \text {, } \\
& \left.g^{\prime} \leqslant e_{1} \text {, by } 2.1 \text { (c) (so, } g^{\prime} \prec e_{1}\right) \\
& \text { (as } f^{\prime} \prec c^{\prime \prime} \equiv t \text { ) } \\
& =a^{\prime}\left(c^{\prime}\left(g^{\prime}\left(w\left(v g^{\prime \prime}\right)\right)\right)\right)=a^{\prime}\left(c^{\prime}\left(g^{\prime}\left((w v) g^{\prime \prime}\right)\right)\right) \text { by (1), (1') } \\
& \text { (as } g^{\prime} \equiv w, v \equiv g^{\prime \prime} \text { ) }
\end{aligned}
$$

further 


$$
\begin{aligned}
& a\left(x b^{\prime \prime}\right)=\left(a^{\prime} c^{\prime}\right)\left(g^{\prime}\left((w v) g^{\prime \prime}\right)\right) \\
& \text { by (11) (as } w \equiv f^{\prime} \equiv v \text {, so } \\
& g^{\prime} \equiv g^{\prime \prime} \equiv w v \in R \text { by }\left(P_{1}\right) \text {, which } \\
& =\left(a^{\prime} c^{\prime}\right)\left(\left(t c^{\prime \prime}\right) b^{\prime \prime}\right) \\
& \text { shows } \left.g^{\prime}\left((w v) g^{\prime \prime}\right) \stackrel{{ }^{\prime}}{\prec} e_{1} \equiv t \equiv c^{\prime}\right) \\
& \text { (as the above shows }\left(t c^{\prime \prime}\right) b^{\prime \prime} \\
& \left.=g^{\prime}\left((w v) g^{\prime \prime}\right)\right) \\
& =\left(h^{\prime}\left(p h^{\prime \prime}\right)\right)\left(\left(t c^{\prime \prime}\right) b^{\prime \prime}\right) \quad \text { where } a^{\prime} c^{\prime}=h^{\prime} p h^{\prime \prime} \text { with } \\
& h^{\prime} \equiv p \equiv h^{\prime \prime} \prec c^{\prime} \text {, by 2.1(a) } \\
& =h^{\prime}\left(\left(p h^{\prime \prime}\right)\left(\left(t c^{\prime \prime}\right) b^{\prime \prime}\right)\right) \quad \text { by (1) }\left(\text { as } h^{\prime} \equiv p \equiv h^{\prime \prime}\right) \\
& =h^{\prime}\left(\left(\left(p h^{\prime \prime}\right)\left(t c^{\prime \prime}\right)\right) b^{\prime \prime}\right) \\
& \text { by }\left(14^{\prime}\right)\left(\text { as } p \equiv h^{\prime \prime} \prec c^{\prime} \equiv t \equiv c^{\prime \prime}\right) \\
& =\left(h^{\prime}\left(\left(p h^{\prime \prime}\right)\left(t c^{\prime \prime}\right)\right)\right) b^{\prime \prime} \\
& \text { by (1) (as 2.1(c') implies } \\
& \left(p h^{\prime \prime}\right)\left(t c^{\prime \prime}\right)=q k^{\prime \prime} \text { with } q \equiv k^{\prime \prime} \equiv h^{\prime \prime} \\
& \equiv h^{\prime} \text {; since } h^{\prime \prime} \prec t \text { ) } \\
& =\left(\left(h^{\prime}\left(p h^{\prime \prime}\right)\right)\left(t c^{\prime \prime}\right)\right) b^{\prime \prime} \\
& \text { by (1) (as } \left.h^{\prime} \equiv p \equiv h^{\prime \prime}\right) \\
& =\left(\left(a^{\prime} c^{\prime}\right)\left(t c^{\prime \prime}\right)\right) b^{\prime \prime} \\
& \text { (as } \left.a^{\prime} c^{\prime}=h^{\prime} p h^{\prime \prime}\right) \\
& =\left(a^{\prime}\left(c^{\prime}\left(t c^{\prime \prime}\right)\right)\right) b^{\prime \prime}=\left(a^{\prime} x\right) b^{\prime \prime} \quad \text { by }(2) \text {, }
\end{aligned}
$$

which proves (18).

5. We can now complete the proof of the Associativity Lemma: i.e. show that (1) to (8') imply the associativity of any product $x y z$. Putting $x=a^{\prime} r a^{\prime \prime}$, $z=b^{\prime} s b^{\prime \prime}$, with $a^{\prime} \equiv r \equiv a^{\prime \prime}, b^{\prime} \equiv s \equiv b^{\prime \prime}$, we have

$$
\begin{aligned}
x(y z) & =\left(a^{\prime}\left(r a^{\prime \prime}\right)\right)\left(y\left(\left(b^{\prime} s\right) b^{\prime \prime}\right)\right) & & \\
& =a^{\prime}\left(\left(r a^{\prime \prime}\right)\left(y\left(\left(b^{\prime} s\right) b^{\prime \prime}\right)\right)\right) & & \text { by }(1)\left(\text { as } a^{\prime} \equiv r \equiv a^{\prime \prime}\right) \\
& =a^{\prime}\left(\left(r a^{\prime \prime}\right)\left(\left(y\left(b^{\prime} s\right)\right) b^{\prime \prime}\right)\right) & & \text { by }\left(1^{\prime}\right)\left(\text { as } b^{\prime} \equiv s \equiv b^{\prime \prime}\right) \\
& =a^{\prime}\left(\left(\left(r a^{\prime \prime}\right)\left(y\left(b^{\prime} s\right)\right)\right) b^{\prime \prime}\right) & & \text { by }\left(16^{\prime}\right)\left(\text { as } r \equiv a^{\prime \prime}\right) \\
& =\left(a^{\prime}\left(\left(r a^{\prime \prime}\right)\left(y\left(b^{\prime} s\right)\right)\right)\right) b^{\prime \prime} & & \text { by }(18) \\
& =\left(a^{\prime}\left(\left(\left(r a^{\prime \prime}\right) y\right)\left(b^{\prime} s\right)\right)\right) b^{\prime \prime} & & \text { by }(17)\left(\text { as } r \equiv a^{\prime \prime}, b^{\prime} \equiv s\right) \\
& =\left(\left(a^{\prime}\left(\left(r a^{\prime \prime}\right) y\right)\right)\left(b^{\prime} s\right)\right) b^{\prime \prime} & & \text { by }(16)\left(\text { as } b^{\prime} \equiv s\right) \\
& =\left(a^{\prime}\left(\left(r a^{\prime \prime}\right) y\right)\right)\left(\left(b^{\prime} s\right) b^{\prime \prime}\right) & & \text { by }\left(1^{\prime}\right)\left(\text { as } b^{\prime} \equiv s \equiv b^{\prime \prime}\right) \\
& =\left(\left(a^{\prime}\left(r a^{\prime \prime}\right)\right) y\right)\left(\left(b^{\prime} s\right) b^{\prime \prime}\right) & & \text { by }(1)\left(\text { as } a^{\prime} \equiv r \equiv a^{\prime \prime}\right) \\
& =(x y) z . & &
\end{aligned}
$$

This completes the proof. 


\section{Applications to regular semigroups.}

1. We saw in $\S 1$ that the multiplication of any regular semigroup $S$ can be described in terms of the partially ordered sets $I=S / R, \Lambda=S / \mathscr{L}$ and the set union of groups $G$, if we know the structure data (Theorem 1): more precisely, all regular semigroups can be described as in Theorem 1, but we still lack necessary and sufficient conditions that the multiplications constructed in that way yield regular semigroups, or, in other words, we still lack a characterization of the structure data of regular semigroups.

We do know some necessary conditions, however. First we know what the structure data must consist of; this must be listed here. First, we must start from sets $I, \Lambda, G$ and an equivalence relation $\equiv$ on the disjoint union $I \cup G \cup \Lambda$; in addition, we know that $I, \Lambda$ carry order relations, and that each class modulo the restriction of $\equiv$ to $G$ is a group; furthermore, each class modulo $\equiv$ intersects $I, G$, and $\Lambda$. By selecting one element $i_{\alpha} \in I$ and one element $\lambda_{\alpha} \in \Lambda$ in each equivalence class (in particular, $i_{\alpha} \equiv \lambda_{\alpha}$ ), we can define relations $\prec$ on $I \times(I \cup G \cup \Lambda), \Lambda \times(I \cup G \cup \Lambda)$ by

$$
i \prec u \Leftrightarrow i \leqslant i_{\alpha} \equiv u, \quad \lambda \prec u \Leftrightarrow \lambda \leqslant \lambda_{\alpha} \equiv u .
$$

We see that $i \prec u \equiv v$ implies $i \prec v, i \leqslant j \prec v$ implies $j \prec v$, and similarly for $\Lambda$. The structure data then consists of this and eleven partial mappings:

$$
\begin{array}{rlrlrl}
(g, m) & \mapsto g m, & G \times I \rightarrow I, & & \text { defined when } m \prec g ; \\
(m, g) & \mapsto g^{m}, & I \times G \rightarrow G, & & \text { defined when } m \prec g ; \\
(g, \nu) & \mapsto \nu g, & G \times \Lambda \rightarrow \Lambda, & & \text { defined when } \nu \prec g ; \\
(\nu, g) & \mapsto g_{\nu}, & \Lambda \times G \rightarrow G, & & \text { defined when } \nu \prec g ; \\
(i, m) & \mapsto i+m, & I \times I \rightarrow I, & & \text { defined when } m \prec i ; \\
(i, m) & \mapsto \sigma_{i, m}, & I \times I \rightarrow G, & & \text { defined when } m \prec i ; \\
(\nu, \lambda) & \mapsto \nu+\lambda, & \Lambda \times \Lambda \rightarrow \Lambda, & & \text { defined when } \nu \prec \lambda ; \\
(\nu, \lambda) & \mapsto v_{\nu, \lambda}, & & \Lambda \times \Lambda \rightarrow I, & & \text { defined when } \nu \prec \lambda ; \\
(i, \lambda) & \mapsto i * \lambda, & I \times \Lambda \rightarrow I, & & \text { always defined; } \\
(i, \lambda) & \mapsto \lambda * i, & I \times \Lambda \rightarrow \Lambda, & & \text { always defined; } \\
(i, \lambda) & \mapsto \tau_{\lambda, i}, & I \times \Lambda \rightarrow G, & & \text { always defined. }
\end{array}
$$

Furthermore, we saw in $\$ 1$ that these maps must always satisfy certain conditions. These are of three kinds. The first type of conditions prescribes compatibility with $\equiv$ and the order relations $\leqslant$; these are:

$$
\begin{aligned}
m & \equiv g^{m} \equiv g m \prec g, & & \text { whenever } m<g ; \\
\nu & \equiv g_{\nu} \equiv \nu g<g, & & \text { whenever } \nu \prec g ;
\end{aligned}
$$




$$
\begin{array}{rlrl}
m & \equiv \sigma_{i, m} \equiv i+m \leqslant i, & \text { whenever } m \prec i ; \\
\nu & \equiv v_{\nu, \lambda} \equiv \nu+\lambda \leqslant \lambda, \quad \text { whenever } \nu \prec \lambda ; \\
i * \lambda & \equiv \tau_{\lambda, i} \equiv \lambda * i, \quad i * \lambda \prec \lambda, \quad \lambda * i \prec i, \quad \text { for all } i, \lambda .
\end{array}
$$

This was seen in 1.4, 1.5, 1.7, 1.8, 1.3. To this we add the following part of 1.9:

$$
\begin{aligned}
& \text { when } j \leqslant i \text {, there exists } m \prec i \text { such that } i+m=j \text {; } \\
& \text { when } \mu \leqslant \lambda \text {, there exists } \nu \prec \lambda \text { such that } \nu+\lambda=\mu \text {. }
\end{aligned}
$$

This says in effect that the order relations on $I, \Lambda$ do not contain more pairs than necessary.

The second group of conditions consists of normalization properties which have also been seen in 1.4, 1.5, 1.7, 1.8, 1.3; in these conditions (and in what follows) the identity elements of the groups which make up $G$ are all denoted by 1 (this creates no confusion, as their location is always clear from context). These conditions are:

$$
\begin{gathered}
1 m=m, 1^{m}=1, \quad \text { when } m \prec 1 ; \quad g i_{\alpha}=i_{\alpha}, g^{i_{\alpha}}=g, \quad \text { when } g \equiv i_{\alpha} ; \\
\nu 1=\nu, 1_{\nu}=1, \quad \text { when } \nu \prec 1 ; \quad \lambda_{\alpha} g=\lambda_{\alpha}, g_{\lambda_{\alpha}}=g, \quad \text { when } g \equiv \lambda_{\alpha} ; \\
i_{\alpha}+m=m, \sigma_{i_{\alpha}, m}=1, i+i_{\alpha}=i, \sigma_{i, i_{\alpha}}=1, \quad \text { when } m<i_{\alpha} \equiv i ; \\
\nu+\lambda_{\alpha}=\nu, v_{\nu, \lambda_{\alpha}}=1, \lambda_{\alpha}+\lambda=\lambda, v_{\lambda_{\alpha}, \lambda}=1, \quad \text { when } \nu<\lambda_{\alpha} \equiv \lambda ; \\
i * \lambda_{\alpha}=i, \tau_{\lambda_{\alpha}, i}=1, \lambda_{\alpha} * i=\lambda_{\beta} \equiv i, \quad \text { when } i \prec \lambda_{\alpha} ; \\
i_{\alpha} * \lambda=i_{\beta} \equiv \lambda, \tau_{\lambda, i_{\alpha}}=1, \lambda * i_{\alpha}=\lambda, \quad \text { when } \lambda \prec i_{\alpha} .
\end{gathered}
$$

The last group consists of the regularity properties established in 1.1:

$$
\begin{gathered}
\text { when } i \equiv i_{\alpha} \text {, some } \lambda \equiv i \text { satisfies } i * \lambda=i_{\alpha}, \lambda * i=\lambda_{\alpha} \\
\text { when } \lambda \equiv \lambda_{\alpha} \text {, some } i \equiv \lambda \text { satisfies } i * \lambda=i_{\alpha}, \lambda * i=\lambda_{\alpha} .
\end{gathered}
$$

2. Conversely, assume that $I, \Lambda, G$ and all the above satisfy these compatibility, normalizaiton and regularity conditions. Let $T=\{(i, g, \lambda) \in I \times G \times$ $\Lambda ; i \equiv g \equiv \lambda\}$. We can try to define a multiplication on $T$ as in Theorem 1; namely, put

$$
(i, g, \lambda)(j, h, \mu)=\left(i+g m, \sigma_{i, g m} g^{m} \tau_{\lambda, j} h_{\nu} v_{\nu h, \mu}, \nu h+\mu\right)
$$

where $m=j * \lambda, \nu=\lambda * j$.

LEMMA 3.1. When the compatibility and normalization conditions hold, the multiplication above on $T$ is well defined. Furthermore, with this multiplication, $T$ is a regular-like groupoid.

Proof. When $(i, g, \lambda),(j, h, \mu) \in T$ and $m=j * \lambda, \nu=\lambda * j$, the compatibility conditions imply: $m \equiv \tau_{\lambda, j} \equiv \nu ; g^{m}$ is defined since $m \prec \lambda \equiv g$, and 
$g^{m} \equiv g m \equiv m$; then $g m \prec i$, so that $i+g m$ and $\sigma_{i, g m}$ are defined and $i+g m \equiv \sigma_{i, g m} \equiv g m \equiv m$. Similarly, $h_{\nu}, v_{\nu h, \mu}$ and $\nu h+\mu$ are defined, and equivalent modulo $\equiv$. In particular, the five group terms lie in the same group (= equivalence class), so that their product is defined and again lies in that same equivalence class. Thus the multiplication on $T$ is well defined.

The verification that $T$ is a regular-like groupoid involves a certain amount of straightforward computations which shall be left to the reader; it is based upon the normalization properties. Define the following subsets of $T$ :

$$
\begin{aligned}
S^{\prime} & =\left\{r_{i}=\left(i, 1, \lambda_{\alpha}\right) ; i \equiv 1 \equiv \lambda_{\alpha}\right\}, \\
R & =\left\{[g]=\left(i_{\alpha}, g, \lambda_{\alpha}\right) ; i_{\alpha} \equiv g \equiv \lambda_{\alpha}\right\}, \\
S^{\prime \prime} & =\left\{q_{\lambda}=\left(i_{\alpha}, 1, \lambda\right) ; i_{\alpha} \equiv 1 \equiv \lambda\right\} .
\end{aligned}
$$

Define $r_{i} \leqslant r_{j}$ by $i \leqslant j, q_{\lambda} \leqslant q_{\mu}$ by $\lambda \leqslant \mu$; we can extend $\equiv$ to $T$ by saying $x \equiv y$ when the components of $x$ are equivalent to those of $y$; and define $r_{i} \prec x$ by $r_{i} \leqslant e_{\alpha} \equiv x, q_{\lambda} \prec x$ by $q_{\lambda} \leqslant e_{\alpha} \equiv x$, where $e_{\alpha}=\left(i_{\alpha}, 1, \lambda_{\alpha}\right)=r_{i_{\alpha}}$ $=[1]=q_{\lambda_{a}}$. Then Axiom (R) holds.

Now take $g \in G, \lambda \in \Lambda, g \equiv \lambda$. We have

$$
[g] q_{\lambda}=\left(i_{\alpha}+g m, \sigma_{i_{\alpha}, g m} g^{m} \tau_{\lambda_{\alpha}, i_{\alpha}} 1_{\nu} v_{\nu 1, \lambda}, \nu 1+\lambda\right) \text {, }
$$

where $g \equiv \lambda \equiv i_{\alpha}, m=i_{\alpha} * \lambda_{\alpha}, \nu=\lambda_{\alpha} * i_{\alpha}$. It follows from the normalization conditions that $m=i_{\alpha}, \nu=\lambda_{\alpha}$, and then $i_{\alpha}+g m=i_{\alpha}, \sigma_{i_{\alpha}, g m}=1, g^{m}=g$, $\tau_{\lambda_{\alpha}, i_{\alpha}}=1,1_{\nu}=1, v_{\nu 1, \lambda}=1$ and $\nu 1+\lambda=\lambda$. Hence, $[g] q_{\lambda}=\left(i_{\alpha}, g, \lambda\right)$.

Similarly, $r_{i}\left([g] q_{\alpha}\right)=(i, g, \lambda)$, whenever $i \equiv g \equiv \lambda$, and this establishes Axiom (D).

The rest of the proof is similar. To verify $(\mathrm{P})$, one uses the normalization conditions to show:

$$
\begin{aligned}
{[g][h] } & =[g h], \quad \text { when } g \equiv h ; \\
q_{\lambda} r_{i} & =\left(i * \lambda, \tau_{\lambda, i}, \lambda * i\right) ; \\
{[g] r_{m} } & =\left(g m, g^{m}, \lambda_{\alpha}\right), \quad \text { when } m \prec g ; \\
q_{\nu}[g] & =\left(i_{\alpha}, g_{\nu}, \nu g\right), \quad \text { when } \nu \prec g ; \\
r_{i} r_{m} & =\left(i+m, \sigma_{i, m}, \lambda_{\alpha}\right), \quad \text { when } m \prec i ; \\
q_{\nu} q_{\lambda} & =\left(i_{\alpha}, v_{\nu, \lambda}, \nu+\lambda\right), \quad \text { when } \nu \prec \lambda ;
\end{aligned}
$$

then $(\mathrm{P})$ follows from the compatibility conditions.

Axiom (E) is similar. Define $E$ to be the set of all $e_{\alpha}$. Then $\left(\mathrm{E}_{1}\right),\left(\mathrm{E}_{2}\right)$ follow from the six formulae above by judicious specialization, while the rest of the axiom is obvious.

In view of this lemma, we can use the Associativity Lemma (Theorem 2) to obtain necessary and sufficient conditions that $T$ be a semigroup. These conditions fall into two groups. Conditions $(4)$ to $\left(8^{\prime}\right)$ (i.e. the associativity in 
cases (4) to $\left(8^{\prime}\right)$ of Theorem 2) will be written later; we note that any two consecutive elements in any of these cases multiply to one of the simple products encountered in $\S 1$, and hence the corresponding associativity conditions are conditions that one would in any case think of considering first; for this reason we call them the obvious associativity conditions.

The remaining conditions, (1) to (3), are trivial, and follow from the normalization properties. This is clear for (3) in view of the formula $[g][h]=$ [ $g h$ ] above. Condition (1) is verified as follows. Take $i \equiv g \equiv \lambda$ and $x=$ $(j, h, \mu) \in T$. Then

$$
\left(r_{i}\left([g] q_{\lambda}\right)\right) x=(i, g, \lambda)(j, h, \mu)=\left(i+g m, \sigma_{i, g m} g^{m} \tau_{\lambda j} h_{\nu} v_{\nu h, \mu}, \nu h+\mu\right),
$$

with $m=j * \lambda, \nu=\lambda * j$. Similarly,

$$
\begin{aligned}
\left([g] q_{\lambda}\right) x & =\left(i_{\alpha}, g, \lambda\right)(j, h, \mu)=\left(g m, g^{m} \tau_{\lambda, j} h_{\nu} v_{\nu h, \mu}, \nu h+\mu\right) \\
& =(g m, k, \nu h+\mu)
\end{aligned}
$$

with $m, \nu$ as above; $i \equiv i_{\alpha}$. When this is multiplied on the left by $r_{i}=$ $\left(i, 1, \lambda_{\alpha}\right)$, one obtains

$$
\left(i+1 n, \sigma_{i, 1 n} i^{n} \tau_{\lambda_{a}, g m} k_{\xi} v_{\xi k, \nu h+\mu}, \xi k+(\nu h+\mu)\right),
$$

with $k$ as above, $n=g m * \lambda_{\alpha}, \xi=\lambda_{\alpha} * g m$. Since $g m \equiv m \prec \lambda \equiv \lambda_{\alpha}$, the normalization properties imply $n=g m, \xi=\lambda_{\beta} \equiv m, 1 n=g m, 1^{n}=1, \tau_{\lambda_{a}, g m}$ $=1, k_{\xi}=1, \xi k=\lambda_{\beta}, v_{\xi k, \nu h+\mu}=1$ and $\xi k+(\nu h+\mu)=\nu h+\mu$ (as $\nu h+\mu \equiv$ $\left.\nu \equiv m \equiv \lambda_{\beta}\right)$. Hence

$$
r_{i}\left(\left([g] q_{\lambda}\right) x\right)=\left(i+g m, \sigma_{i, g m} k, h+\mu\right)=\left(r_{i}\left([g] q_{\lambda}\right)\right) x .
$$

Condition (2) is verified similarly, and $\left(1^{\prime}\right),\left(2^{\prime}\right)$ follow by duality.

We can now state

THEOREM 3. With I, G, $\Lambda$ as above, assume that the compatibility, normalization, regularity and obvious associativity conditions are satisfied. Let $T=$ $\{(i, g, \lambda) \in I \times G \times \Lambda ; i \equiv g \equiv \lambda\}$ with the multiplication

$$
(i, g, \lambda)(j, h, \mu)=\left(i+g m, \sigma_{i, g m} g^{m} \tau_{\lambda, j} h_{\nu} v_{\nu h, \mu}, \nu h+\mu\right),
$$

where $m=j * \lambda, \nu=\lambda * j$. Then $T$ is a regular semigroup. Furthermore, the structure data of $T$ is precisely the given data. Conversely, every regular semigroup can be constructed in that fashion.

Proof. The converse is Theorem 1; hence we only have to continue the proof. The results obtained so far show that, under the hypothesis of the theorem, $T$ satisfies all the requirements of the Associativity Lemma, and so is a semigroup. [This is by far the sharpest part of the theorem.] We now turn to regularity. 
When $x=(i, g, \lambda) \in T$, put $R(x)=i$. When $z \in T$, then $R(x z)=i+g m$ for some $m \prec i$ and, hence, $R(x z) \leqslant R(x)$. Conversely, assume $R(y) \leqslant$ $R(x)$; say, $y=(j, h, \mu), j \leqslant i$. We shall show that this implies $y=x z$ for some $z \in T$; more precisely, we shall show that $r_{i} \in x T$ and $y \in r_{i} T$.

By the regularity conditions, there exists $p \in I$ such that $\lambda \equiv p, p * \lambda=i_{\alpha}$, $\lambda * p=\lambda_{\alpha}$, where $i \equiv g \equiv \lambda \equiv i_{\alpha} \equiv \lambda_{\alpha}$. With $u \in G, u \equiv \lambda_{\alpha}$, we have

$$
(i, g, \lambda)\left(p, u, \lambda_{\alpha}\right)=\left(i+g m, v_{i, g m} g^{m} \tau_{\lambda, p} u_{\nu} v_{v u, \lambda_{\alpha}}, v u+\lambda_{\alpha}\right) \text {, }
$$

where $m=p * \lambda=i_{\alpha}, \nu=\lambda * p=\lambda_{\alpha}$; hence the normalization conditions imply $i+g m=i, \sigma_{i, g m}=1, g^{m}=g, u_{v}=u, v_{v u, \lambda_{\alpha}}=1$ and $\nu u+\lambda_{\alpha}=\lambda_{\alpha}$. Hence $(i, g, \lambda)\left(p, u, \lambda_{\alpha}\right)=\left(i, g \tau_{\lambda, p} u, \lambda_{\alpha}\right)$; in the group of all $u \in G$ with $u \equiv i_{\alpha}$ we can find one $u$ such that $(i, g, \lambda)\left(p, u, \lambda_{\alpha}\right)=r_{i}$. Thus $r_{i} \in x T$.

Considering now $y=(j, h, \mu)$ with $j \leqslant i$, we first have $j=i+m$ for some $m \prec i$, by the compatibility condition; there $m \equiv j \equiv h \equiv \mu$. Taking $v \in G$, $v \equiv h$, we have (using the associativity in $T$ )

$$
r_{i} r_{m}[v] q_{\mu}=r_{i+m}\left[\sigma_{i, m}\right][v] q_{\mu}=\left(i+m, \sigma_{i, m} v, \mu\right) ;
$$

we can always find $v$ so that this is $y$, and so $y \in r_{i} T$. [This can also be shown as we did for $r_{i} \in x T$, without implicitly using the associativity.]

Thus we have shown that $y \in x T$ if and only if $R(y) \leqslant R(x)$. In particular, $x \Re y$ if and only if $R(x)=R(y)$; and $T / R \cong I$ (as partially ordered sets). Dually, $T / \mathcal{L} \cong \Lambda$. If now we take $i \in I, \lambda \in \Lambda$ such that $i \equiv \lambda$, $i * \lambda=i_{\alpha}(\equiv i), \lambda * i=\lambda$, then we see, as in the proof of 1.12 , that $(i, u, \lambda)$ is an idempotent of $T$ for some $u$ (the calculation is the same, of course). The regularity conditions then show that every $R$-class of $T$ (and, every $\mathcal{L}$-class) contains an idempotent; and $T$ is a regular semigroup.

We also have some information about the structure data of $T$. Up to the obvious isomorphisms and identifications, $I$ and $\Lambda$ are as given, which immediately implies that $x \equiv y$ if and only if $x \mathscr{D} y$, so that $e_{\alpha}$ is a suitable choice of idempotents and $G$ is as given (identifying $g$ and $[g]$ ). That this yields for $T$ the very some structure data as we started with (up to these isomorphisms and identifications) is then obvious on the formulae $q_{\lambda} r_{i}=$ $r_{i * \lambda}\left[\tau_{\lambda, i}\right] q_{\lambda, i}$, etc., established before.

3 . Theorem 3 is, strictly speaking, a full structure theorem for regular semigroups, which constructs them in terms of groups and partially ordered sets, by means of mappings satisfying explicit conditions. However, as a structure theorem, it leaves much to be desired, since it needs no less than eleven actions and factor sets, satisfying seven compatibility conditions, six normalization conditions, the two regularity conditions, and the nine obvious associativity conditions, some of which we shall see are rather technical. Forthcoming papers will show that part of the trouble is inadequate presentation, namely there are better ways to present the operations + and $*$ and the 
partial actions of $G$ on $I$ and $\Lambda$, than by giving a longish list of technical conditions. At any rate, Theorem 3 is not likely to be very useful as a structure theorem.

All the same it gives us extremely valuable new information about regular semigroups.

Many classes of regular semigroups have been constructed. These constructions are mostly in terms of one $\Re$-class (partial semigroup), for the many bisimple examples, or the band of idempotents, in the orthodox cases; constructions in terms of groups and partially ordered sets are obviously more desirable, but not so common. Theorem 3 shows that this last type of construction, the type that describes most of the structure, is possible in general.

In addition, the theorem also tells us how to obtain such a construction; and, by showing that only the obvious associativity conditions need be considered, it takes care of the most difficult part of the construction, leaving only parts which we have seen are entirely straightforward. Thus it should prove a most valuable method of constructing regular semigroups.

We shall see in the next section that this method is still useful in the case of other types of constructions. The essential part of the theorem is, of course, the most difficult: that is, the Associativity Lemma.

4. We complete this section by giving the nine obvious associativity conditions, with various comments and an example showing their possible use. The reader must keep in mind that the fact that only these conditions must be considered is rather more important than the conditions themselves.

The nine conditions are obtained, either by straightforward calculation, or by means of the formulae $q_{\lambda} r_{i}=r_{i{ }_{\lambda}}\left[\tau_{\lambda, i}\right] q_{\lambda, i}$, etc. (then using associativity in cases (1) to (3)). These calculations are left to the reader. Each condition splits into three conditions that hold in $I, G, \Lambda$ respectively, some of which are trivial. The conditions are:
(4I) $(g h) m=g(h m)$,
(4G) $(g h)^{m}=g^{h m} h^{m}, \quad$ where $m \prec g \equiv h ;$
$\left(4_{\Lambda}^{\prime}\right) \nu(g h)=(\nu g) h$,
$\left(4_{G}^{\prime}\right) \quad(g h)_{\nu}=g_{\nu} h_{\nu g}, \quad$ where $\nu \prec g \equiv h$;
(5I) $g m * \nu=g_{\nu}(m * \nu g)$,
$\left(5_{\Lambda}\right) \quad \nu g * m=(\nu * g m) g^{m}$,
$\left(5_{G}\right) \tau_{\nu, g m}\left(g^{m}\right)_{\nu * g m}=\left(g_{\nu}\right)^{m * \nu g} \tau_{\nu g, m}$, where $\nu \prec g, m \prec g$;
(6 $) \quad g(m+n)=g m+g^{m} n$,
$\left(6_{G}, \quad g^{m+n} \sigma_{m, n}=\sigma_{g m, g^{m}}\left(g^{m}\right)^{n}, \quad\right.$ where $n \prec m \prec g$;
$\left(6_{\Lambda}^{\prime}\right)(\xi+\nu) g=\xi g_{\nu}+\nu g$,
$\left(6_{G}^{\prime}\right) \quad v_{\xi, \nu} g_{\xi+\nu}=\left(g_{v}\right)_{\xi} v_{\xi g_{\nu}, \nu g}, \quad$ where $\xi \prec \nu \prec g$;
$\left(7_{I}\right) m+(n+p)=(m+n)+\sigma_{m, n} p$, 


$$
\begin{aligned}
& \left(7_{G}\right) \quad \sigma_{m, n+p} \sigma_{n, p}=\sigma_{m+n, \sigma_{m, n} p}\left(\sigma_{m, n}\right)^{p}, \quad \text { where } p \prec n \prec m ; \\
& \left(7_{\Lambda}^{\prime}\right) \quad(\xi+\nu)+\lambda=\xi v_{\nu, \lambda}+(\nu+\lambda), \\
& \left(7_{G}^{\prime}\right) \quad v_{\xi, \nu} v_{\xi+\nu, \lambda}=\left(v_{\nu, \lambda}\right)_{\xi} v_{\xi v_{\nu, \lambda}, \nu+\lambda}, \quad \text { where } \xi \prec \nu \prec \lambda ; \\
& \left(8_{I}\right) \quad(i+m) * \lambda=(i * \lambda)+\tau_{\lambda, i}(m *(\lambda * i)), \\
& \left(8_{G}\right) \quad \tau_{\lambda, i+m}\left(\sigma_{i, m}\right)_{\lambda *(i+m)}=\sigma_{i * \lambda, \tau_{\lambda, i}(m *(\lambda * i))}\left(\tau_{\lambda, i}\right)^{m *(\lambda * i)} \tau_{\lambda * i, m}, \\
& \left(8_{\Lambda}\right) \quad(\lambda * i) * m=(\lambda *(i+m)) \sigma_{i, m}, \quad \text { where } m \prec i ; \\
& \left(8_{I}^{\prime}\right) \quad(i * \lambda) * \nu=v_{\nu, \lambda}(i *(\nu+\lambda)), \\
& \left(8_{G}^{\prime}\right) \quad\left(v_{\nu, \lambda}\right)^{i *(\nu+\lambda)} \tau_{\nu+\lambda, i}=\tau_{\nu, i}\left(\tau_{\lambda, i}\right)_{\nu *(i * \lambda)} v_{(\nu *(i * \lambda)) \tau_{\lambda, i} \lambda * i}, \\
& \left(8_{\Lambda}^{\prime}\right) \quad(\nu+\lambda) * i=(\nu *(i * \lambda)) \tau_{\lambda, i}+(\lambda * i), \quad \text { where } \nu \prec \lambda .
\end{aligned}
$$

These formulae carry implications that both sides are defined in the indicated circumstances; for instance, $\left(8_{I}\right)$ carries the implication that, when $m \prec i$, then also $m *(\lambda * i) \prec i * \lambda$.

A good case can be made that, despite appearances, these associativity conditions are the very simplest that can be given in this situation. One sees that each expresses a compatibility relationship between two of the structure data maps: e.g. $\left(4_{I}\right)$ relates the multiplication in $G$ with its action on $I$, etc. It is clear that all these relationships must be accounted for, and very doubtful that in this general situation any of them is a consequence of the others. In addition, they were derived from so-called simple products, which do indeed give the simplest multiplications (the reader should convince himself of that fact by calculating products such as $r_{i} r_{j}, g r_{j}$, without the hypothesis that $j \prec i, j \prec g$ ).

Nevertheless we see on these conditions that the usefulness of Theorem 3 as a structure theorem for arbitrary regular semigroups is necessarily limited. If, indeed, conditions (4), (6), (7) and their duals are satisfactory, however, the conditions tying the star operations do not give a very adequate way of coping with these operations in general; it can be seen at the beginning of $\S 1$ that these operations must be investigated more thoroughly than we have done; also, a certain amount of complex structure is still hidden in these operations, for instance the location of the idempotents of $S$ in $I \times \Lambda$, so that they are, in effect, possibly the most important part of the structure data.

There are, however, some situations where Theorem 3 can be used efficiently. The first case is when the subgroups of $S$ are trivial; in this case, conditions (4) to $\left(8^{\prime}\right)$ simplify sufficiently to give adequate descriptions of all the structure data, including the star operations. As an example of this, we consider the case where $S$ is a bisimple semigroup with identity and trivial group of units, of type $\omega$; i.e. $S / R=I$ is the descending chain of integers 
$0>1>2>\cdots>n>n+1>\cdots$ (the usual order on integers is used hereafter).

In this case, we select $i_{0}=0$, and $\lambda_{0} \in \Lambda$ arbitrary. We note that $i * \lambda_{0}=i$ for all $i$, by the normalization conditions; this implies that $i_{0}$ is the only $i$ with $i * \lambda_{0}=i_{0}$, in particular, the $\mathcal{L}$-class $\lambda_{0}$ contains only one idempotent; since $\lambda_{0}$ was arbitrary, it follows that $S$ is a left inverse semigroup. For each $\lambda$ there is, therefore, precisely one $\lambda \delta \in I$ such that $\lambda \delta * \lambda=0, \lambda * \lambda \delta=\lambda_{0}$; this defines a mapping $\delta: \Lambda \rightarrow I$. We now investigate the + and $*$ operations, noting that by Theorem 3 we can write $S$ as $I \times \Lambda$ with multiplication given by $(i, \lambda)(j, \mu)=(i+(j * \lambda),(\lambda * j)+\mu)$, with + and $*$ defined everywhere.

Conditions (7) show that both + operations are associative. We remember from 1.9 that $m \mapsto i+m$ is an isomorphism of $I$ upon $\{i, i+1, \ldots\}$; since there is only one such isomorphism, the operation + on $I$ is the usual addition of nonnegative integers. Dually, $\Lambda$ is a right cancellative semigroup under + . [It is easily seen that $\Lambda$ is just the $R$-class of the identity element $\left(0, \lambda_{0}\right)$ of $S$. Furthermore, 1.9 need not be quoted, because by Theorem 3 the conditions on the structure data will imply that fact: hence we can also verify it directly from the conditions.] Now define a mapping $\theta: \Lambda \rightarrow \Lambda$ by $\lambda \theta=$ $\lambda * 1$. It follows from $\left(8_{\Lambda}\right)$ that $\lambda * i=\lambda \theta^{i}$ for all $i$ (with $\lambda * 0=\lambda \theta^{0}=\lambda$ by the normalization conditions). On the other hand, $\lambda \delta * \lambda=0$, and then $\left(8_{I}\right)$ shows $i * \lambda=0$ whenever $i \leqslant \lambda \delta$, whereas $(\lambda \delta+k) * \lambda=0+\left(k * \lambda_{0}\right)=k$ whenever $k \geqslant 0$. From these descriptions it is then easy to translate the associativity conditions into conditions on $\delta, \theta$; one finds that $\delta$ must be a homomorphism, $(\lambda+\mu) \theta=\lambda+\mu \theta$ whenever $\mu \neq \lambda_{0}$ (with $\lambda_{0} \theta=\lambda_{0}$ ), and $(\lambda \theta) \delta=\lambda \delta-1$ when $\lambda \neq \lambda_{0}$. This provides an alternate proof for the structure theorem of these semigroups (Corollary 2.2 of [2]).

A specially important instance of the case when all subgroups of $S$ are trivial is when $S$ is a band. This is of importance because orthodox semigroups are frequently constructed in terms of their band of idempotents, but the structure of general bands is still far from being well understood. The later refinements of this theory will bring no further light on that case, so that Theorem 3 is so far the best that can be obtained by our methods. It is hoped that a later paper will discuss that case in more detail.

The last case of interest is that of orthodox semigroups. In this case, the star operations can be described in terms of the multiplication of idempotents: if $i \in I, \lambda \in \Lambda$, then there are idempotents $e=(j, g, \lambda), f=(i, h, \mu)$ and knowledge of ef allows the description of $i * \lambda, \tau_{\lambda, i}$ and $\lambda * i$ in terms of the rest of the structure data. In the general case, one can see that this description is sufficiently complicated that it makes a poor structure theorem. Still, it shows that orthodox semigroups can be explicitly constructed in terms of groups and their band of idempotents; the knowledge that such constructions exist is not to be neglected, even when the theoretical construction is 
impracticable in that situation, as shown by the coextension theory; for it is a good inducement to find a practicable construction. In one case, inverse semigroups, the remark above does yield to a practicable construction, which is the subject of a forthcoming paper.

\section{REFERENCES}

1. G. R. Baird, On semigroups and uniform partial bands, Semigroup Forum 4 (1972), 185-188. MR 45 \#415.

2. A. H. Clifford, The structure of bisimple left unipotent semigroups as ordered pairs, Semigroup Forum 5 (1972/73), 137-144. MR 48 \#11365.

3. A. H. Clifford and G. B. Preston, The algebraic theory of semigroups, Vol. 1, Math. Surveys, no. 7, Amer. Math. Soc., Providence, R. I., 1961. MR 24 \# A2627.

4. Nicolas Farès, Idempotents et 1 -classes dans les demigroups et les anneaux, C. R. Acad. Sci. Paris Sér. A-B 269 (1969), A341-A343. MR 40 \# 2777.

5. P. A. Grillet, Left coset extensions, Semigroup Forum (to appear).

6. A A coherence theorem for Schützenberger groups, J. Austral. Math. Soc. (submitted).

7. J. E. Leech, Two papers: $\mathcal{X}$-coextensions of monoids, and The structure of a band of groups, Mem. Amer. Math. Soc. No. 157 (1975).

Department of Mathematics, Tulane University, New Orleans, Louisiana 70118 